\title{
THE BOLOCAM GALACTIC PLANE SURVEY: SURVEY DESCRIPTION AND DATA REDUCTION
}

\author{
James E. Aguirre ${ }^{1}$, Adam G. Ginsburg ${ }^{2}$, Miranda K. Dunham ${ }^{3}$, Meredith M. Drosback $^{4}$, John Bally $^{2}$, Cara Battersby $^{2}$, \\ Eric Todd Bradley ${ }^{5}$, Claudia Cyganowski ${ }^{6}$, Darren Dowell ${ }^{7}$, Neal J. Evans II ${ }^{3}$, Jason GlenN $^{2}$, Paul Harvey $^{2,3}$, \\ Erik Rosolowsky ${ }^{8}$, Guy S. Stringfellow ${ }^{2}$, Josh Walawender ${ }^{9}$, and Jonathan P. Williams ${ }^{10}$ \\ ${ }^{1}$ Department of Physics and Astronomy, University of Pennsylvania, Philadelphia, PA, USA; jaguirre @ sas.upenn.edu \\ ${ }^{2}$ CASA, University of Colorado, 389-UCB, Boulder, CO 80309, USA \\ ${ }^{3}$ Department of Astronomy, University of Texas, 1 University Station C1400, Austin, TX 78712, USA \\ ${ }^{4}$ Department of Astronomy, University of Virginia, P.O. Box 400325, Charlottesville, VA 22904, USA \\ ${ }^{5}$ Department of Physics, University of Central Florida, 4000 Central Florida Boulevard, Orlando, FL 32816-2385, USA \\ ${ }^{6}$ Department of Astronomy, University of Wisconsin, Madison, WI 53706, USA \\ ${ }^{7}$ Jet Propulsion Laboratory, California Institute of Technology, 4800 Oak Grove Drive, Pasadena, CA 91104, USA \\ ${ }^{8}$ Department of Physics and Astronomy, University of British Columbia, Okanagan, Canada \\ ${ }^{9}$ Institute for Astronomy, University of Hawaii, 640 North Aohoku Place, Hilo, HI 96720, USA \\ ${ }^{10}$ Institute for Astronomy, University of Hawaii, 2680 Woodlawn Drive, Honolulu, HI 96822, USA \\ Received 2009 June 29; accepted 2010 September 15; published 2010 December 16
}

\begin{abstract}
We present the Bolocam Galactic Plane Survey (BGPS), a $1.1 \mathrm{~mm}$ continuum survey at 33" effective resolution of $170 \mathrm{deg}^{2}$ of the Galactic Plane visible from the northern hemisphere. The BGPS is one of the first large area, systematic surveys of the Galactic Plane in the millimeter continuum without pre-selected targets. The survey is contiguous over the range $-10.5 \leqslant l \leqslant 90.5,|b| \leqslant 0.5$. Toward the Cygnus X spiral arm, the coverage was flared to $|b| \leqslant 1.5$ for $75.5 \leqslant l \leqslant 87.5$. In addition, cross-cuts to $|b| \leqslant 1.5$ were made at $l=3,15,30$, and 31 . The total area of this section is $133 \mathrm{deg}^{2}$. With the exception of the increase in latitude, no pre-selection criteria were applied to the coverage in this region. In addition to the contiguous region, four targeted regions in the outer Galaxy were observed: IC1396 $\left(9 \mathrm{deg}^{2}, 97.5 \leqslant l \leqslant 100.5,2.25 \leqslant b \leqslant 5.25\right)$, a region toward the Perseus Arm ( $4 \mathrm{deg}^{2}$ centered on $l=111$, $b=0$ near NGC 7538), W3/4/5 (18 $\left.\mathrm{deg}^{2}, 132.5 \leqslant l \leqslant 138.5\right)$, and Gem OB $1\left(6 \mathrm{deg}^{2}, 187.5 \leqslant l \leqslant 193.5\right)$. The survey has detected approximately 8400 clumps over the entire area to a limiting non-uniform $1 \sigma$ noise level in the range 11-53 mJy beam ${ }^{-1}$ in the inner Galaxy. The BGPS source catalog is presented in a previously published companion paper. This paper details the survey observations and data reduction methods for the images. We discuss in detail the determination of astrometric and flux density calibration uncertainties and compare our results to the literature. Data processing algorithms that separate astronomical signals from time-variable atmospheric fluctuations in the data timestream are presented. These algorithms reproduce the structure of the astronomical sky over a limited range of angular scales and produce artifacts in the vicinity of bright sources. Based on simulations, we find that extended emission on scales larger than about 5.9 is nearly completely attenuated (>90\%) and the linear scale at which the attenuation reaches $50 \%$ is 3'8. Comparison with other millimeter-wave data sets implies a possible systematic offset in flux calibration, for which no cause has been discovered. This presentation serves as a companion and guide to the public data release (http://irsa.ipac.caltech.edu/Missions/bolocam.html) through NASA's Infrared Processing and Analysis Center (IPAC) Infrared Science Archive (IRSA). New data releases will be provided through IPAC-IRSA with any future improvements in the reduction. The BGPS provides a complementary long-wavelength spectral band for the ongoing ATLASGAL and Herschel-SPIRE surveys, and an important database and context for imminent observations with SCUBA-2 and ALMA.
\end{abstract}

Key words: ISM: clouds - methods: data analysis - stars: formation - stars: massive - submillimeter: ISM surveys

Online-only material: color figures

\section{INTRODUCTION}

Millimeter-wavelength continuum surveys of the Galactic plane provide the most efficient way to find molecular clumps that are the likely formation sites of massive stars and star clusters. The development of detector arrays has made blind surveys of large areas possible. Such surveys bypass the need for selection based on the presence of embedded stars or star clusters, infrared sources, masers, or radio continuum emission. In particular, they can locate molecular clumps before stars form, providing vital information on the initial conditions of star formation. These surveys can also provide valuable constraints on the physical properties of the clumps, especially masses and mean densities, when combined with distance information.
Galaxy-wide surveys are essential for measuring the impacts of the environment on clump properties and star formation activity. Do clump properties vary with Galactocentric distance, or with location with respect to spiral arms? Do they depend on the level of nearby star formation activity? Answering these questions in our Galaxy will provide the essential "ground truth" required for the analysis of distant galaxies where individual clouds and clumps are not resolved and only galaxy-wide average quantities can be measured.

Surveys of the galaxy in low-lying rotational transitions of the CO molecule (Dame et al. 2001; Jackson et al. 2006) have identified the locations of large molecular clouds and traced the transition from atomic gas in the outer galaxy to molecular gas in the inner galaxy. Surveys in $\mathrm{CO}$ have detected 
a strong preference for giant molecular clouds to form along spiral arms (Stark \& Lee 2006). Recent improved measurements of kinematic distances (e.g., Pohl et al. 2008) and accurate distances from VLBI (Reid et al. 2009) have refined our view of the spiral structure of the Milky Way. This new increase in data now allows a more complete investigation of the relationship between spiral density waves and star formation.

Locating the early stages of star formation is quite difficult and often relies on the serendipitous location of a cold, dark cloud which appears as an infrared dark cloud (IRDC). While dark clouds were known from optically obscured regions, the "discovery" of IRDCs from the Midcourse Space Experiment $(M S X)$ satellite was presented by Egan et al. (1998) and Carey et al. (1998). IRDCs have now been extensively cataloged with MSX (Simon et al. 2006a) and GLIMPSE $8 \mu \mathrm{m}$ data (Peretto $\&$ Fuller 2009). In the past decade, compelling evidence has emerged which suggests that IRDCs are the pre-cursors to massive stars, and therefore, stellar clusters (see, e.g., Rathborne et al. 2006, 2008). IRDCs, however, require the favorable viewing condition of being in front of a bright, mid-IR background for detection. Millimeter wave surveys, however, provide an efficient means of identifying cold, dense dust throughout the Galaxy independent of the Galactic background, and thus may be seen at large distances. Moreover, the millimeter-wave emission is optically thin, allowing the properties of the cloud to be determined. Millimeter wave surveys such as BGPS are essential for understanding massive star and cluster formation on a Galactic scale, from the inner to the outer Galaxy.

Studies of nearby clouds have demonstrated that star formation is far from uniform over molecular clouds, but is concentrated in unusually dense regions called clumps or cores, which fill a small fraction of the area of most clouds (Lada et al. 1991; Enoch et al. 2007; Evans et al. 2009). Following Williams et al. (2000) and McKee \& Ostriker (2007), we use the term "core" to refer to a very dense region destined to form an individual star or small multiple star system and the term "clump" to refer to a region likely to form a group or cluster of stars. While clumps forming massive clusters may be even denser than cores forming low mass stars (Mueller et al. 2002), in general, clumps are larger and have a lower mean density (McKee \& Ostriker 2007).

To delineate clumps from their parent clouds as traced in $\mathrm{CO}$, line emission from molecules which are high-density tracers, such as $\mathrm{NH}_{3}, \mathrm{H}_{2} \mathrm{CO}, \mathrm{CS}, \mathrm{HCN}$, etc., are extremely useful. Spectral lines also provide excellent diagnostics of lineof-sight motions, temperatures, and densities. However, the interpretation of these gas tracers is complicated by variations in tracer abundances caused by freezeout onto grains, sublimation caused by star formation, and subsequent complex chemical processing (van Dishoeck \& Blake 1998). Interpretation is further complicated by uncertainties in optical depths and excitation conditions and the impacts of radiation fields and shocks. These complications make the derivation from such molecular line data of column densities, masses, and other physical properties of star-forming clumps very difficult. A further limitation of spectral line surveys is that the tracers of denser gas tend to be weak, and multi-element receiver arrays have only a modest number of elements, making blind surveys very time consuming. Instruments are beginning to be developed to address this limitation, such as NRAO's $K$-band Focal Plane Array ${ }^{11}$ and JCMT's HARP-B (Buckle et al. 2009).
In contrast, focal-plane arrays containing hundreds of individual bolometers sensitive to millimeter and submillimeter (sub$\mathrm{mm}$ ) radiation are now available. This advance in technology enables blind surveys of the Galactic plane, providing a uniform inventory of massive star-forming and starless clumps. Catalogs resulting from these surveys will provide the database for subsequent observations in tracers such as hard-to-excite molecular lines and higher resolution studies with instruments such as CARMA and ALMA. A significant advantage of continuum observations of dust at long wavelengths is the low optical depth of the dust (Johnstone \& Bally 2006), permitting a straightforward estimate of the mass of the emitting region.

Submillimeter observations of Galactic sources have been conducted by a variety of different groups focusing on small regions. The Submillimeter Common-User Bolometer Array (SCUBA; Holland et al. 1999) on the $15 \mathrm{~m}$ James Clerk Maxwell Telescope mapped many significant regions in the Galactic plane over its eight year lifetime (see Di Francesco et al. 2008 for a summary), but never completed a contiguous survey of the Galactic plane. The Bolocam instrument has been used on the $10.4 \mathrm{~m}$ Caltech Submillimeter Observatory $\left(\mathrm{CSO}^{12}\right)$ to map nearby large molecular clouds at $1.1 \mathrm{~mm}$ and identify hundreds of cores (Enoch et al. 2007). MAMBO on the $30 \mathrm{~m}$ IRAM telescope has been used at $1.2 \mathrm{~mm}$ to map both small, nearby cores (Kauffmann et al. 2008) and more distant regions of more massive star formation (Rathborne et al. 2006; Motte et al. 2007). None of these surveys covered a significant fraction of the Galactic plane.

This situation has begun to change dramatically. Recent widearea surveys have been carried out in the submillimeter bands 250-500 $\mu \mathrm{m}$ by the Balloon-borne Large Aperture Submillimeter Telescope (Olmi et al. 2009; Roy et al. 2010; Netterfield et al. 2009; Chapin et al. 2008). This work provides an unprecedented view of a wide range of star-forming environments and demonstrates the richness of submillimeter data sets, creating great anticipation for the imminent results from instruments aboard Herschel, particularly the SPIRE camera. From the ground, the ATLASGAL survey (Schuller et al. 2009), using the LABOCA instrument at $870 \mu \mathrm{m}$ with $19^{\prime \prime} .2$ resolution on the APEX telescope in Chile, has covered $95 \mathrm{deg}^{2}$ in the Galactic Plane, $-30 \leqslant l \leqslant 11.5$ and $15 \leqslant l \leqslant 21$ with $|b| \leqslant 1$. The survey will eventually encompass $-60 \leqslant l \leqslant 60$ with $|b| \leqslant 1.5$.

The BGPS is the first millimeter survey of a substantial fraction of the Galactic Plane in the northern hemisphere. The BGPS maps in the first quadrant overlap with the VLA Galactic Plane Survey (VGPS) in H I and radio continuum (Stil et al. 2006), the Spitzer-GLIMPSE and GLIMPSE-II fields (Benjamin et al. 2003), MIPSGAL (Carey et al. 2009), the BU-FCRAO ${ }^{13} \mathrm{CO} J=1 \rightarrow 0$ Galactic Ring Survey (Jackson et al. 2006), and ATLASGAL (Schuller et al. 2009), among others. The BGPS coverage will also overlap with the upcoming Herschel HiGAL (Molinari et al. 2010) and SCUBA-2/JCMT Plane Surveys (Di Francesco 2008). The BGPS provides a long-wavelength dust continuum data point, complementing the spectral energy distribution (SED) provided by other surveys. By covering both the Galactic center and anti-center regions with a single instrument, a consistent comparison over the widest possible range of Galactocentric radius is possible. The BGPS gives a view of massive star and cluster formation throughout the Galaxy and provides an important finder chart for future

\footnotetext{
12 The Caltech Submillimeter Observatory is supported by the NSF.
} 


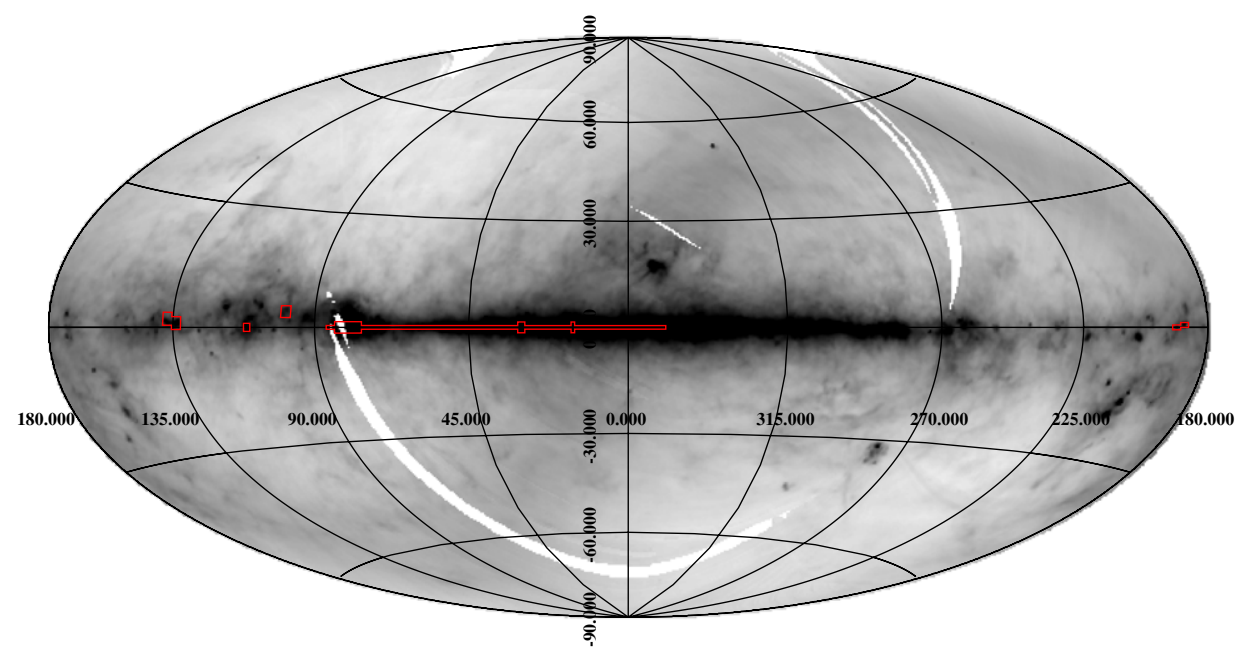

Figure 1. Coverage of the BGPS, showing the continuous coverage in the first quadrant, and the regions targeted in the outer Galaxy. The background gray scale is IRAS $100 \mu \mathrm{m}$.

(A color version of this figure is available in the online journal.)

high-resolution observations with facilities such as CARMA and ALMA.

This paper presents the imaging data from the Bolocam Galactic Plane Survey (BGPS). The source catalog is described in a previously published companion paper (Rosolowsky et al. 2010; hereafter R10). The outline of this paper is as follows. Section 2 describes the instrument and the observations. The data analysis is described in Sections 3 (astrometry), 4 (the mapmaking algorithm), and 5 (flux calibration). Key discussions are in Section 5.3, which explains the effect of the data processing on the accurate recovery of extended structure, and in Section 5.5, which compares the BGPS flux with other surveys, including a discussion of a possible systematic offset. The final image data products and the public release are described in Section 6. We conclude in Section 7 with a brief discussion of the broad features of the BGPS in comparison with other surveys.

\section{OBSERVATIONS}

We used Bolocam ${ }^{13}$ to survey more than $170 \mathrm{deg}^{2}$ of the northern Galactic Plane. Bolocam is the facility 144 element bolometer array camera mounted at the Cassegrain focus of the $10.4 \mathrm{~m}$ mirror of the CSO on the summit of Mauna Kea. We used the filter configuration with a band center of $271.1 \mathrm{GHz}$ (hereafter $1.1 \mathrm{~mm})$ and fractional bandwidth $\Delta v / v=0.17(46 \mathrm{GHz})$. The detectors are silicon nitride micromesh absorbers with neutron transmutation doped germanium thermistors, operated at a temperature of $\sim 250 \mathrm{mK}$. The Bolocam array field of view (FOV) is 7.5, with individual detectors having nearly Gaussian beams of $31^{\prime \prime}$ FWHM. The spacing between the individual detector beams on the sky is $38^{\prime \prime}$, so the focal plane is not instantaneously fully sampled. The Bolocam instrument is described in greater detail in Glenn et al. (2003).

The maps presented here were acquired during six separate observing sessions at the CSO over the course of two years between 2005 June and 2007 September. The observing epochs are given in Table 1. Bolocam observations were typically scheduled when $\tau_{225}>0.06$. Between observing epochs, Bolocam was removed from its mount at the re-imaged Cassegrain focus and stored warm. Thus, the flux calibration and pointing model

\footnotetext{
13 http://www.cso.caltech.edu/bolocam
}

Table 1

Observing Epochs for the BGPS

\begin{tabular}{lll}
\hline \hline Number & Begin (UT) & End (UT) \\
\hline I & 2005 Jul 3 & 2005 Jul 9 \\
II & 2005 Sep 5 & 2005 Sep 12 \\
III & 2006 Jun 2 & 2006 Jun 30 \\
IV & 2006 Sep 3 & 2006 Sep 19 \\
V & 2007 Jul 1 & 2007 Jul 25 \\
VI & 2007 Sep 4 & 2007 Sep 9 \\
\hline
\end{tabular}

were re-computed for each epoch to allow for variations in the instrument and optics. The final data products were aligned to the well-constrained pointing model created from the Epoch $\mathrm{V}$ and VI data (Section 3). We found that the flux calibration did not in fact differ significantly between epochs; see Section 5.1 for more details.

Figure 1 shows the coverage of the BGPS. The survey is contiguous over the range $-10.5 \leqslant l \leqslant 90.5,|b| \leqslant 0.5$. Toward the Cygnus $\mathrm{X}$ spiral arm, the coverage was flared to $|b| \leqslant 1.5$ for $75.5 \leqslant l \leqslant 87.5$. In addition, cross-cuts to $|b| \leqslant 1.5$ were made at $l=3,15,30$, and 31 . The total area of this section is $133 \mathrm{deg}^{2}$. With the exception of the increase in latitude, no pre-selection criteria were applied to the coverage in this region. In addition to the contiguous region, four targeted regions in the outer Galaxy were observed: IC1396 $\left(9 \mathrm{deg}^{2}, 97.5 \leqslant l \leqslant 100.5,2.25 \leqslant b \leqslant 5.25\right)$, a region toward the Perseus Arm (4 $\mathrm{deg}^{2}$ centered on $l=111, b=0$ near NGC 7538), W3/4/5 (18 $\left.\mathrm{deg}^{2}, 132.5 \leqslant l \leqslant 138.5\right)$, and Gem OB1 $\left(6 \mathrm{deg}^{2}, 187.5 \leqslant l \leqslant 193.5\right)$. The total area of good coverage for the BGPS is $170 \mathrm{deg}^{2}$.

Our basic observing strategy was to raster scan a field by moving the primary mirror of the CSO. This was done to modulate the astrophysical signal faster than fluctuations in atmospheric opacity. Each field was scanned in a pattern with alternating rasters along lines of constant $l$, followed by a series of rasters along lines of constant $b$. Fields were observed several times with such raster scans to improve signal-to-noise ratio $(\mathrm{S} / \mathrm{N})$. Thus, each point in the final map of a field was observed multiple times with the array moving in different directions with respect to the astrophysical emission, helping to separate it from 

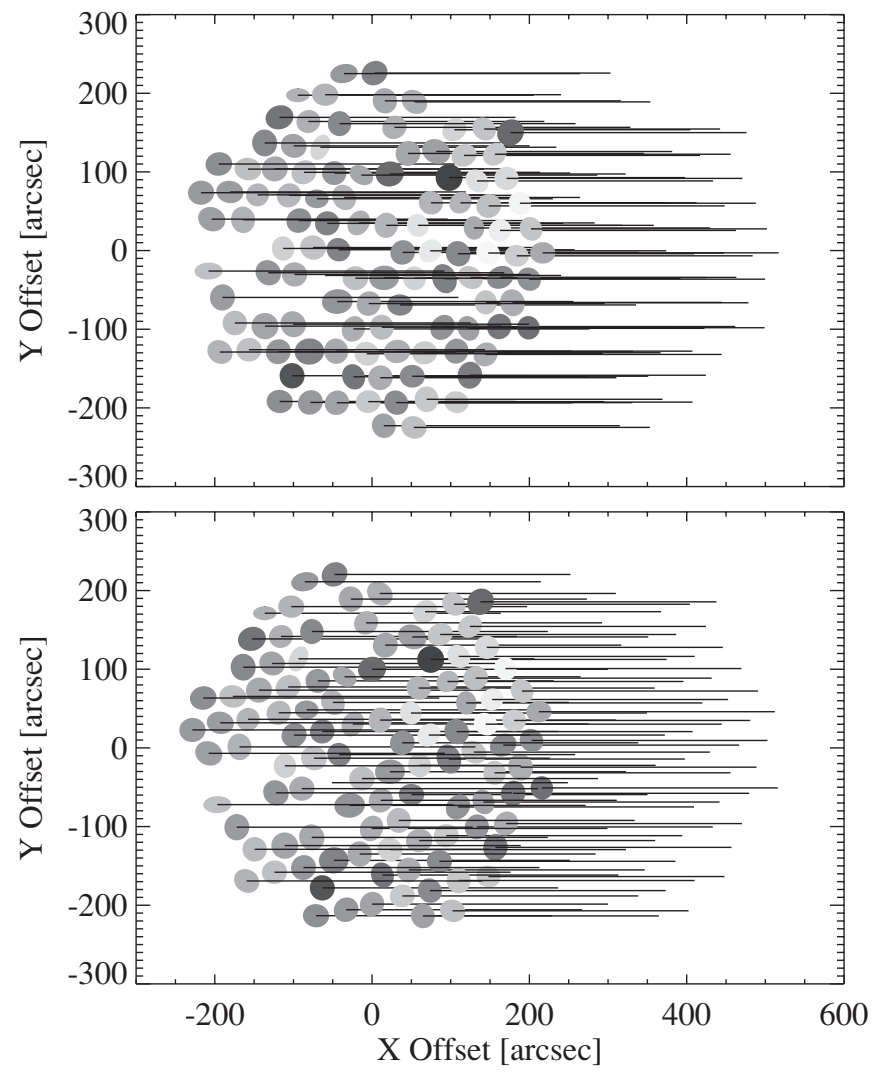

Figure 2. Top: the position and ellipticity of the detectors in the Bolocam focal plane as mapped to the sky. The size of the pixel corresponds to its FWHM; it is clear that the beams do not completely overlap on the sky. The relative response to source is shown by the gray scale, with the darkest being the largest response. The path of the center of each pixel as the array is scanned from left to right is shown for a particular orientation of the array relative to the scan direction. In this case, the sampling of the sky orthogonal to the scan direction is poor. Bottom: the same as the top, but with the array rotated to its optimal angle relative to the scan direction. It is clear that the sampling orthogonal to the scan direction is now much more uniform.

atmospheric emission (see Section 4 for further details on this process).

In Epochs I and II, the fundamental observing block was a region $1^{\circ} \times 1^{\circ}$, covered by 23 rasters along each of $l$ or $b$. Starting with Epoch III, the fundamental block was changed to $3^{\circ} \times 1^{\circ}$ for increased mapping efficiency (i.e., less time spent in turnarounds at the end of a raster). Each $3^{\circ} \times 1^{\circ}$ block was covered using either 23 rasters along lines of constant $b$, or 67 rasters along lines of constant $l$. In both cases, the spacing between adjacent rasters was $162^{\prime \prime}$. The total time for observing the entire block was 39 (48) minutes for rasters along constant $b(l)$. The data were electronically sampled at $10 \mathrm{~Hz}$ along the scan direction, slightly higher than the Nyquist rate for the scan speed of $120^{\prime \prime} \mathrm{s}^{-1}$.

These fundamental observing blocks were stitched together to make 29 large, contiguous maps. These images define the "fields" used for comparison. Each "field" includes all observations covering a region between 1 and $6 \mathrm{deg}^{2}$.

Because the Bolocam detector beams do not fully overlap on the sky, if the array is scanned along detector rows, gaps will be left in the resulting map. To ameliorate this, we began using in Epoch II a field rotator to adjust the rotation angle of the array with respect to the scan direction so that as the scan proceeded the full extent of the FOV in the direction

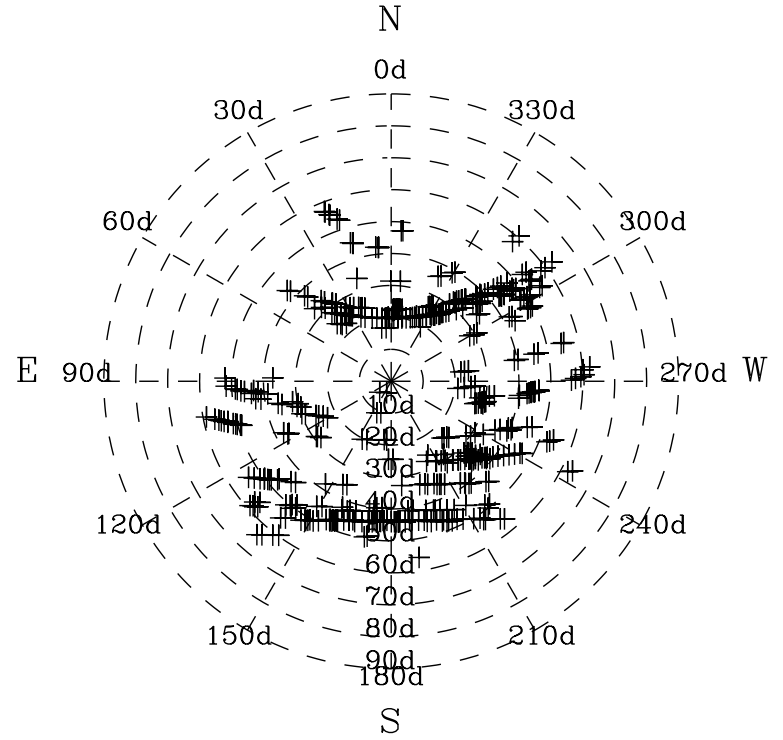

Figure 3. Distribution of the pointing calibration sources across the local sky in Hawaii during 2007 July (Epoch V) when the master pointing model was constructed. Note that the entire local sky is sampled, and the residual pointing rms is valid for any point on the sky.

orthogonal to the scan was sampled by at least one detector. This is shown in Figure 2. Achieving a uniform sampling along the scan-orthogonal direction is further complicated by a number of missing bolometers. A simulation was performed to determine the optimal angle to rotate the array with respect to the scan direction to account for both the effects of the beam spacing and missing bolometers. During the turnaround following a raster, the field rotator is adjusted to this optimum angle for the subsequent raster. Without the field rotator, the coverage shows variations of $100 \%$ from pixel to pixel in a single raster of a field. With the rotator, this is reduced to $\sim 40 \%$.

\section{ASTROMETRY}

\subsection{Absolute Reference Sources and Pointing Model}

We constructed an absolute reference system for the BGPS by observing bright quasars and blazars near the Galactic Plane with small raster maps. The sources were chosen from the SMA Submillimeter Calibrator List, ${ }^{14}$ since they have positions reliably determined from interferometric measurements and are point sources at the scale of the Bolocam beam. The distribution of absolute pointing sources over the sky is shown in Figure 3.

Pointing observations were performed approximately once every $2 \mathrm{hr}$ over each night. The elevation offsets showed a deviation from zero which was empirically well modeled by a quadratic function of altitude. No systematic deviation was observed for the azimuth offsets. The derived model is shown in Figure 4. An rms scatter of $\sim 6^{\prime \prime}$ for the model over the entire observed range was achieved, with somewhat worse scatter in altitude than in azimuth.

\subsection{Pixel Positions in the FOV}

In addition to the model for the pointing center, it is necessary to empirically determine the actual projected pattern of the array on the sky and measure the rotation angle between the focal

\footnotetext{
$\overline{14}$ http://sma1.sma.hawaii.edu/callist/callist.html
} 


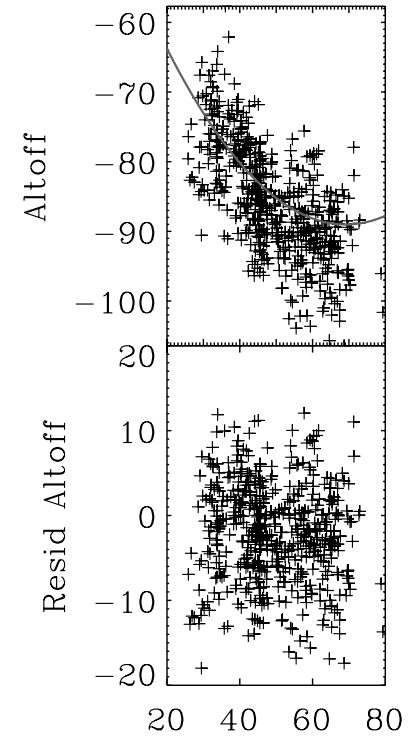

(a)
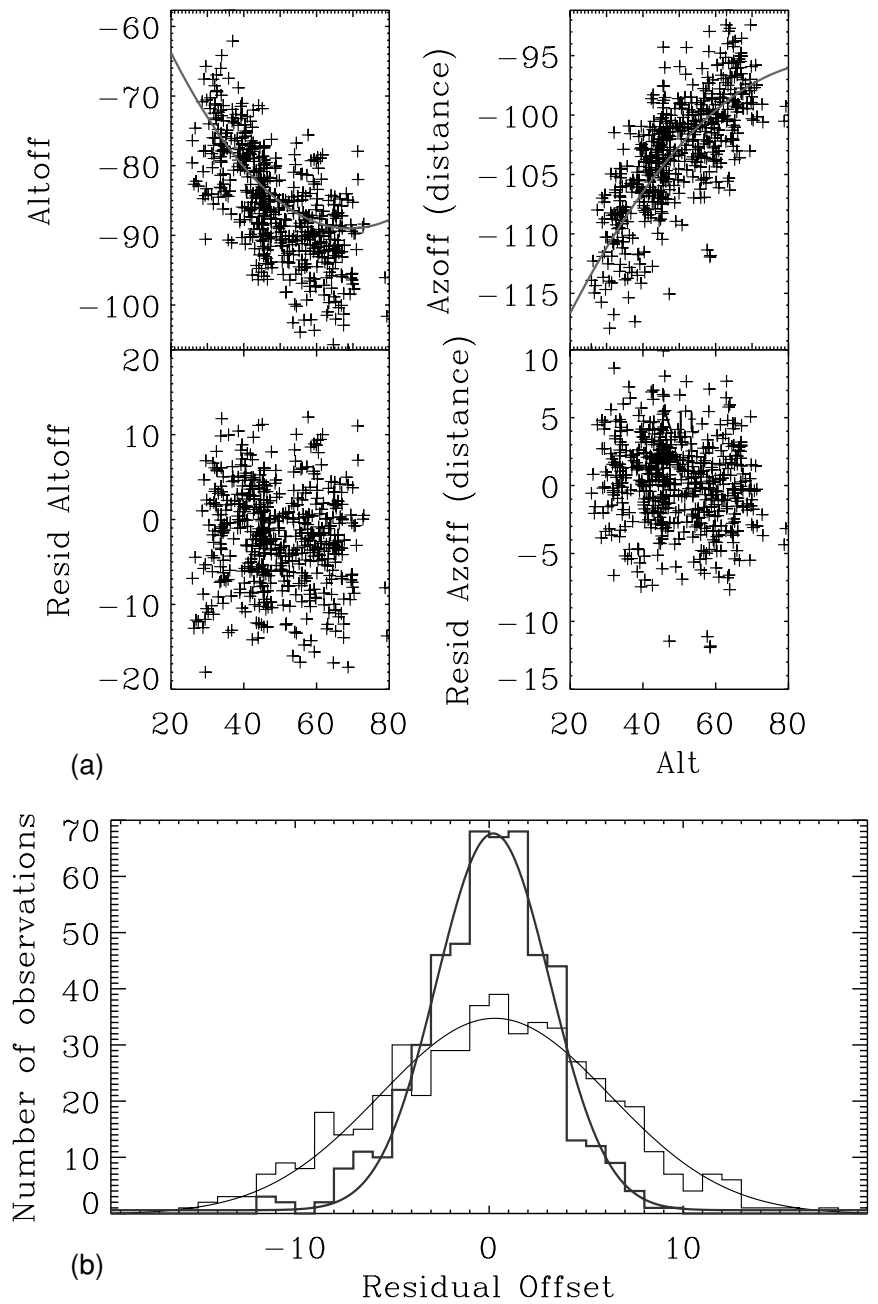

Figure 4. Bolocam pointing model for Epoch V. (a) The pointing model for Epoch V, from which the master reference images were derived for subsequent alignment. The left column is the pointing model correction and the right the residuals (both in arcseconds). The red line indicates the fitted model. Five hundred and ten pointing sources were included, and the final rms was 5".77 in altitude and 3". 21 in azimuth, or a total rms offset of 6.' 6 . No systematic offset with azimuth was found. (b) The residuals of the pointing model. The Gaussian fits have $\sigma=5.94,2.92$ in altitude offset and azimuth offset, respectively.

plane and the sky coordinate system. This is done by making observations that track all detectors across a bright source (e.g., a planet) and making maps from each bolometer individually.

In our first data release, the projected positions of the detectors on the sky are made using the nominal positions without correction for optical distortion. This results in a slightly reduced effective resolution ( $33^{\prime \prime}$ instead of $\left.31^{\prime \prime}\right)$. The effective beam size was measured from fully sampled beam-mapping observations of planets and is a good description of the blurring of the beam for the portions of a field observed by all the detectors. Within about one FOV of the field edges, however, only a subset of the array observes the field, and so the effective beam size varies slightly at these positions, and may be asymmetric. Because this distortion is asymmetric, it can also result in a small pointing offset. However, this effect is strongly mitigated by the low number of hits at field edges: these data are generally flagged out. The residual offset is found to be within the overall pointing model error, but caution should be used when measuring source locations at the field edges in the first data release. Future releases will have the distortion correction applied, removing this effect and improving the effective resolution.

\subsection{Relative Alignment and Mosaicking}

A master pointing field was constructed from observations taken in the epoch with the best-constrained pointing model for that field (either Epoch V or VI) for every field observed by the BGPS. Relative alignment between the master and other observations of the same field was performed by finding the peak of the cross-correlation between images. The cross-correlation peak was fit with a Gaussian profile and the difference between the Gaussian peak and the image center was used as the pointing offset. The offset may be determined accurately to within the error in finding the peak of the Gaussian, typically $<1^{\prime \prime}$. To create the final image, all observations of a field were merged into a single timestream with these pointing offsets applied.

This method of alignment makes use of all the information available in the maps, and avoids the ambiguities inherent in using extracted sources to align fields, as the BGPS sources are rarely pointlike. It further avoids the slightly larger effective beam and loss of peak flux density which would result if the maps of individual observations were combined using the pointing model alone, where each individual observation would be co-added with the $6^{\prime \prime}$ rms uncertainty of the model. In some fields with few sources, particularly those in the range $l=65-75$, there was not enough signal to acquire a pointing offset using cross-correlation. In these fields, the pointing model was used directly, and so the above-mentioned effects affect these fields.

\subsection{Comparison to the SCUBA Legacy Catalog}

All SCUBA 850 and $450 \mu \mathrm{m}$ data have been re-processed in a uniform manner and made publicly available as the SCUBA Legacy Catalog (Di Francesco et al. 2008, hereafter SLC). These maps allow a cross-check of the accuracy of the BGPS pointing model. We have applied the same cross-correlation procedure used to obtain the relative alignment of BGPS observations to compare the BGPS to the SLC $850 \mu \mathrm{m}$ images. Di Francesco et al. (2008) claim a typical pointing accuracy for SCUBA of $\sim 3^{\prime \prime}$, with offsets as large as $\sim 6^{\prime \prime}$ occurring occasionally. No further adjustment to the nominal JCMT pointing model was performed in producing the SLC maps, and thus we assume these numbers are typical.

There are a number of differences in the Bolocam and SLC maps which might lead to difficulties in comparing their astrometry. In particular, different scan strategies were used (SCUBA observations were typically taken in jiggle-map mode, which tends to remove extended structure), and of course there is the difference in wavelength and beam size between the instruments. Nevertheless, the by-eye morphological comparison between SLC $850 \mu \mathrm{m}$ and BGPS sources is generally excellent, as exemplified in Figure 5(a). A cross-correlation technique was used in order to minimize these limitations, since it does not require the establishment of a single position for an object.

The SLC has the advantage of having observations over a large portion of the plane, allowing the pointing to be checked (in spots) for deviations as a function of position. To test this, 21 of the 29 BGPS fields were used which overlapped with the SLC. For these fields, we find $\Delta l=1^{\prime \prime} .8 \pm 1^{\prime \prime} .2, \Delta b=-0.4 \pm 0$.' 8 , nearly consistent with zero, and an rms dispersion about the mean BGPS position of 6.2 , consistent with the errors derived for the Bolocam pointing model. The offset for each field is shown in Figure 5(b). We have not corrected the Bolocam pointing based on the SLC or any external data set, but have produced our final maps with the internally derived model described in Section 3.1. 


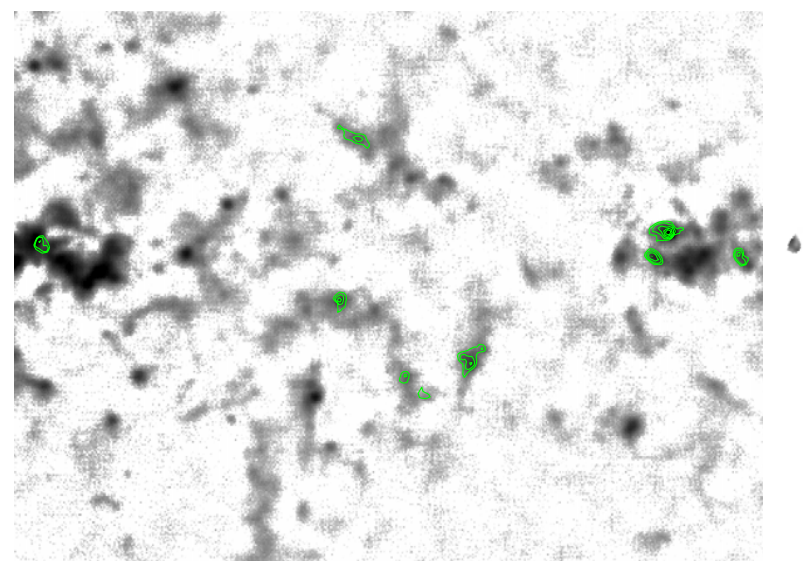

(a)

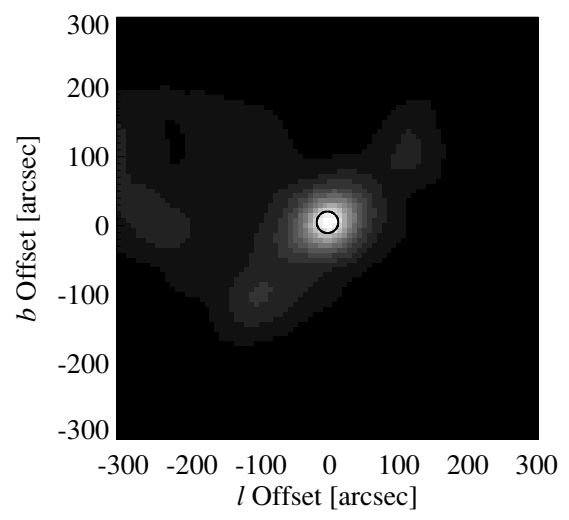

(b)

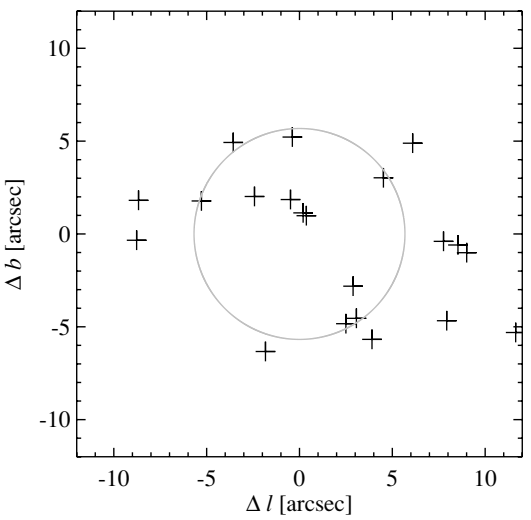

(c)

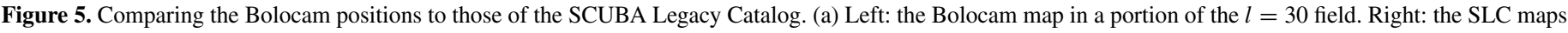

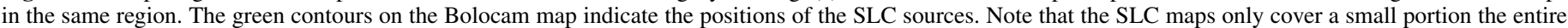

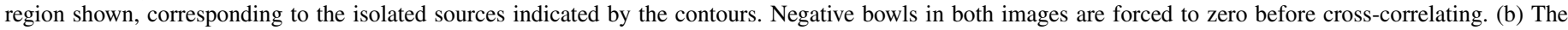

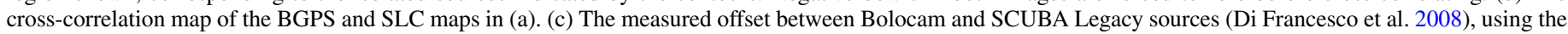
method described in Section 3.4. The circle indicates the $1 \sigma$ region, which is also consistent with the error derived from the internal Bolocam pointing model.

(A color version of this figure is available in the online journal.)

\section{MAPPING ALGORITHM}

At wavelengths near $1 \mathrm{~mm}$, the emission from the atmosphere dominates any astrophysical emission. For an atmospheric zenith optical depth of $\tau=0.1$, the sky brightness temperature is around $30-40 \mathrm{~K}$ at typical zenith angles. This background loading determines the instrument calibration (see Section 5.1), but the primary time-variable signal is due to few percent fluctuations in atmospheric opacity. The conversion $J$ from $\mathrm{Jy} \mathrm{beam}^{-1}$ to Kelvin for Bolocam is

$$
J=1 \times 10^{26} \frac{2 k}{A_{\text {eff }}}=58 \frac{\mathrm{Jy} \mathrm{beam}^{-1}}{\mathrm{~K}},
$$

where $k$ is Boltzmann's constant and $A_{\text {eff }}$ is the effective collecting area of the telescope. Thus, atmosphere fluctuations are tens of $\mathrm{Jy} \mathrm{beam}^{-1}$, as compared to the typical brightness of Galactic sources, which lie in the range of $0.1-1 \mathrm{Jy}^{\text {beam }}{ }^{-1}$. The essential signal-processing problem to be solved by the mapping algorithm, then, is the estimation of the astrophysical emission in the presence of the fluctuating atmosphere signal, without a priori knowledge of either.

For this work, a custom pipeline was developed to address issues specific to the BGPS. It is based on, but significantly improves, the method of Enoch et al. (2006) for generating maps and characterizing their properties, and is similar in many respects to the methods used by other bolometer cameras, e.g., Cotton et al. (2009; MUSTANG/GBT) or Kovács (2008; SHARC-2/CSO). It also incorporates some of the technical developments of Sayers et al. (2009) developed for Bolocam data at $2.1 \mathrm{~mm}$. A key element of the reduction is the iterative estimation of the atmospheric fluctuations and the astrophysical signal. The atmospheric model is developed from a set of principal components of the bolometer signal time series under the assumption that the bulk of the correlated signal is atmospheric. After subtraction of the atmosphere model, the astrophysical emission is estimated, then subtracted, and the process is repeated.

\subsection{Algorithm}

We assume the raw timestream data $d$ for each bolometer (indexed by $i$ ) at discrete time points (indexed by $t$ ) can be written as

$$
d_{i t}=s_{i t}+a_{i t}+c_{i t}+e_{i t}+p_{i t}+\varepsilon_{i t},
$$

where $s$ is the astrophysical signal, $a$ is the median atmospheric fluctuation seen by all detectors, $c$ are terms correlated between bolometers that are distinct from $a, e$ are non-random signals due to the instrument itself, $p$ are long timescale fluctuations not 
modeled by $a$ or $c$, and $\varepsilon$ is irreducible Gaussian noise due to photon fluctuations and detector noise. (Each of these terms is discussed in greater detail in the following sections.)

In the presence of purely Gaussian noise, the maximum likelihood map $m$ can be obtained by minimizing the goodnessof-fit statistic

$$
\chi^{2}=(d-A m)^{T} W(d-A m) .
$$

Here $d$ is the concatenated data from all bolometers (of dimen$\operatorname{sion} N_{\text {tod }}=$ number time samples of all detectors). $A$ provides the mapping between a given sample in the time-ordered data and the pixel in the sky map to which it corresponds. $A$ is a matrix of dimension $N_{\text {tod }} \times N_{\text {pix }}=$ number of map pixels. Each element of $A$ is either 1 or 0 . Note that we are considering the data $d$ and the map $m$ as vectors (the mapping to a two-dimensional map being implicit in the matrix $A$ ). $W$ is an $N_{\text {tod }} \times N_{\text {tod }}$ matrix which is the inverse of the covariance matrix $N$ of the time domain noise $\varepsilon$,

$$
N_{t t^{\prime}}=\left\langle\varepsilon_{t} \varepsilon_{t^{\prime}}\right\rangle,
$$

where \langle\rangle denotes an ensemble average over many realizations of the random timestream noise process $\varepsilon_{t}$. Further detail about the way in which $W$ was estimated is given in Section 4.6.

The result of the $\chi^{2}$ minimization is

$$
m=\left(A^{T} W A\right) A^{T} W d .
$$

Thus, the mapping from data to map is a linear operator, $M=\left(A^{T} W A\right) A^{T} W$. For compactness, Equation (5) will be written as $M[d]=m$. Note that the mapping operator is not invertible. However, given a map $m$ and an observing matrix $A$, there exists a linear operation which makes predictions about the observed timestreams, namely $d=A m$; this will be denoted by $T[m]=d$.

Because the model $A m$ in Equation (3) only includes the celestial contribution to the observed data $d$, direct use of Equation (5) will produce a map containing artifacts due to the unmodeled components $a, c, e$, and $p$. One approach would be to include templates for these terms as additional rows in $A$ and proceed with a simultaneous fit. In general, however, the correct forms to use are not known a priori. The goal, then, is to produce a time series for each bolometer which as closely as possible approximates $s+\varepsilon$ so that we may produce the best estimate of the astrophysical signal $m=M[s+\varepsilon]=S+N$. We proceed iteratively, estimating each term in Equation (2) in the order of its relative strength. In the following, we denote the best estimate of a time series $x$ in the $n$th iteration as $\tilde{x}^{(n)}$.

In the first iteration, we estimate the largest signal, $a$, according to the method in Section 4.2, and assume $\tilde{s}_{i t}^{(0)}=$ $\tilde{c}_{i t}^{(0)}=\tilde{p}_{i t}^{(0)}=0$. We then form

$$
\xi_{i t}^{(n)}=d_{i t}-\tilde{a}_{i t}^{(n)}-\tilde{c}_{i t}^{(n)}-\tilde{e}_{i t}^{(n)}-\tilde{p}_{i t}^{(n)} \approx s_{i t}+\varepsilon_{i t} .
$$

This is the best estimate of signal plus irreducible noise at iteration step $n$ and is made into a map

$$
M\left[\xi_{i t}^{(n)}\right]=\tilde{m}^{(n)} .
$$

The current best map $\tilde{m}^{(n)}$ is then deconvolved to provide a relatively low-noise, smooth map from which to generate a timestream

$$
T\left[\mathcal{D}\left[\tilde{m}^{(n)}\right]\right]=\tilde{s}_{i t}^{(n)},
$$

where $\mathcal{D}$ represents the deconvolution operation. At this stage, we can subtract both $\tilde{a}_{i t}^{(n)}$ and $\tilde{s}_{i t}^{(n)}$ from the original data and estimate $\tilde{c}_{i t}^{(n)}, \tilde{e}_{i t}^{(n)}$, and $\tilde{p}_{i t}^{(n)}$. The iterative process begins again with Equation 6. An example time series showing the successive removal of these models is shown in Figure 6. We discuss the estimation of each of the various terms $a, c, e$, and $p$ in the following sections.

It is useful at each step to produce a "residual map"

$$
E=M\left[d_{i t}-\tilde{s}_{i t}^{(n)}-\tilde{a}_{i t}^{(n)}-\tilde{c}_{i t}^{(n)}-\tilde{e}_{i t}^{(n)}-\tilde{p}_{i t}^{(n)}\right] .
$$

This residual map serves as a visualization of the progress of the iterative method, since in a perfect process it would be map of $\varepsilon$.

\subsection{Atmosphere Fluctuation Model}

Since atmosphere fluctuations are the largest signal in the raw data, they are modeled first. By construction, the Bolocam beams as they pass through the dominant layer of atmospheric water vapor a few kilometers above the telescope are still highly overlapped, and therefore sample a nearly identical region of atmosphere. The simplest model for the atmosphere fluctuations makes use of this fact by assuming that the largest correlated signals between detectors are due to the atmosphere fluctuations; this is the term $a$ in Equation (2). We construct a model of the atmosphere fluctuations which are seen in common by all detectors, which we denote the "median atmosphere model." In each iteration $n$, we construct the non-atmosphere-fluctuation part of the time series as

$$
\tilde{a}_{i t}^{(n)}=d_{i t}-\tilde{s}_{i t}^{(n)}-\tilde{c}_{i t}^{(n)}-\tilde{e}_{i t}^{(n)}-\tilde{p}_{i t}^{(n)} .
$$

The median atmosphere model is then simply

$$
\tilde{a}_{t}^{(n)}=\operatorname{median}_{i}\left[\tilde{a}_{i t}^{(n)}\right] .
$$

Each bolometer's timestream is then fit to the median atmosphere model, i.e., we find $r_{i}^{(n)}$ such that $\tilde{a}_{i t}^{(n)}$ most closely approximates $r_{i}^{(n)} \tilde{a}_{t}^{(n)}$. This defines the relative gains of the detectors as

$$
r_{i}^{(n)}=\frac{\sum_{t^{\prime}} \tilde{a}_{i t^{\prime}}^{(n)} \tilde{a}_{t^{\prime}}^{(n)}}{\sum_{t^{\prime}}\left(\tilde{a}_{t^{\prime}}^{(n)}\right)^{2}}
$$

The $r_{i}$ converge fairly rapidly. (See the discussion in Section 5.1.)

The simple common mode model of Equation (11) does not remove all of the signal correlated at zero time lag between the bolometers. Some of this remaining correlated signal may also be atmosphere. Sayers et al. (2010) found for the $2.1 \mathrm{~mm}$ Bolocam data that the subtraction of a second-order spatial polynomial across the array accounted for nearly all of their residual noise; however, the $1.1 \mathrm{~mm}$ data seem to have additional correlated components that are not completely described by such a model. Therefore, to further remove the atmosphere (and any other correlated instrument noise) without a detailed physical model, we use a principal component analysis (PCA) as given in Laurent et al. (2005). While this method was developed for point source detection in deep, high-redshift surveys, the technique works equally well for this survey, with the caveat that it removes large-scale structure on the size of the array FOV. This effect is mitigated by the iterative process (the Laurent et al. 2005 analysis did not iterate). Figure 7 shows an example of the modes removed by the PCA model. These are a graphical visualization of the terms $c$ in Equation (2). 


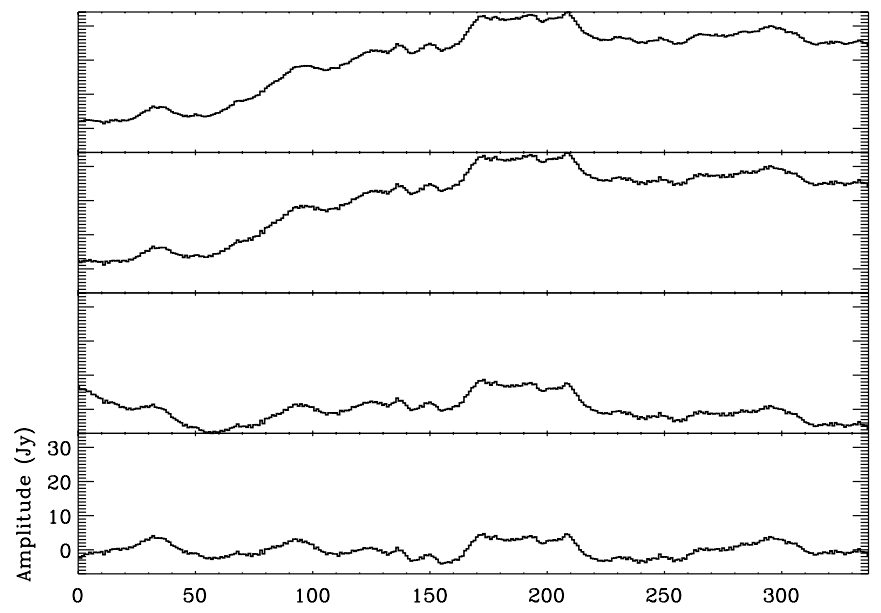

\subsection{Instrument Error Signals}

Most of the instrument error signals have characteristic features which aid in their removal. In particular, unlike the atmosphere emission and correlated signals described above, these signals are not correlated between detector channels. The error signals include the following.

1. Pickup from the $60 \mathrm{~Hz} \mathrm{AC} \mathrm{power.} \mathrm{This} \mathrm{appears} \mathrm{as} \mathrm{narrow}$ lines in the power spectral density of the data. The second harmonic of $60 \mathrm{~Hz}$ is aliased via beating against the $130 \mathrm{~Hz}$ bolometer bias frequency into $10 \mathrm{~Hz}$ with sidebands split at $\sim 1 \mathrm{~Hz}$. It is removed by replacing components at these frequencies in the Fourier transform with Gaussian noise matched to the local mean amplitude.

2. Spikes in voltage due to cosmic ray strikes on the bolometers ("glitches"). An example of a glitch in the time series is shown in Figure 8, along with the distribution of glitch amplitude extracted.

3. Microphonic pickup due to vibrations of the receiver. The microphonic effect is due to a change in the capacitive coupling of the readout wires to the circuit ground, which is converted by the high impedance of the detectors into a measurable signal, much like a condenser microphone. The most noticeable microphonics occur at the end of each scan when the telescope is turning around and the field rotator is adjusting. This leads to broad spikes in the time series, whose long decay must be removed from the data, particularly during the beginnings of scans.

Fortunately, most of the AC powerline pick-up occurs at frequencies where there is no astrophysical signal, given the beam size and scan speed (cf. Figure 1 of Sayers et al. 2009). The out-of-band lines are dealt with by low-pass filtering the data, and the remaining in-band lines are replaced with Gaussian noise as indicated above. Because of the low correlation with astrophysical signal, this step is performed only once and is not iterated.

Both glitches and microphonic pickup from the scan turnarounds are degenerate with astrophysical signal and must be estimated as part of the iterative process. Glitches are identified as large excursions from the rms level after subtraction of the best atmosphere and bright source model, and the data there are excluded in subsequent maps. The turnaround microphonics are explicitly modeled as decaying exponentials at the beginnings and ends of scans. Residuals from this model may also be removed by the PCA cleaning.

After iteratively subtracting the astrophysical model, each scan also has a fifth-order polynomial fit in time removed to deal with the longest time scale modes ( $p$ in Equation (2)). These modes are not correlated between detectors. They correspond to spatial scales much larger than those to which Bolocam remains sensitive after subtraction of the PCA model, so their subtraction produces maps with fewer "stripes," without appreciably degrading the signal.

\subsection{Data Flagging}

Due to the large volume of data generated by the survey, it was necessary to develop new tools to quickly visualize the data and ensure data quality. A common tool in radio astronomy is the so-called waterfall plot, which is an image with frequency on the $x$-axis, time on the $y$, and an intensity proportional to the interferometer visibility amplitude or phase. We have used a variant on this to visualize the Bolocam data by displaying 

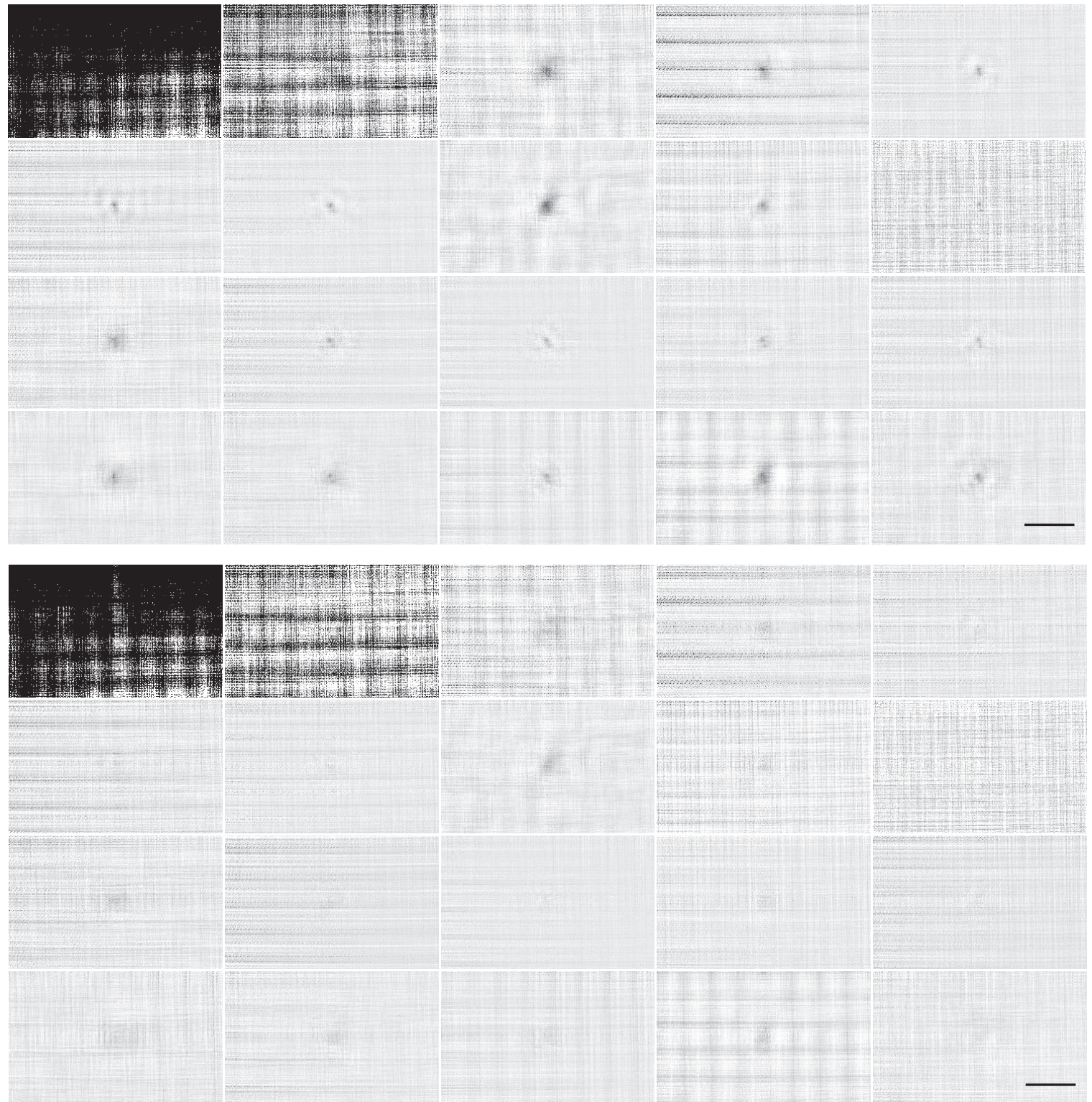

Figure 7. Each PCA component in time can be turned into a map. Above are selected images (in inverted gray scale) to illustrate the PCA components and the effect of iteration on the process of creating these components. The displays are in Galactic coordinates with a color range from -0.1 to $1.0 \mathrm{Jy}^{\mathrm{b}}$ beam ${ }^{-1}$. The bar in the bottom right indicates a $10^{\prime}$ length. Top four rows: a grid of maps of each of the first 20 PCA components displayed at the same scale. It is clear that NCG 7538, the object imaged, has emission in each component. There are varying levels of noise in each component, with the first and second being the most obvious atmospheric components. Most of the other noise components are probably detector noise correlated among a subset of the bolometers. The streaks are residuals of the scan-turnaround noise that was not removed by the exponential model fit. Bottom four rows: the same figure, but after 20 iterations. The figure is the breakdown of the atmosphere remainder (i.e., the raw data minus the astrophysical model) into eigenfunctions. Very little astrophysical flux remains at any level of correlation, though there is some at large (few arcminute) scales.

bolometer number (related to the bolometer position in the focal plane) on the $x$-axis and time on the $y$, with the intensity given by the bolometer's response (in Jy) at that time. An example of this is shown in Figure 9. Anomalies such as the glitch shown can be detected manually (with practice) and interactively flagged out.

An automated flagger was also created that flags out outlier data on a per-map-pixel basis. In order to make a robust measurement of the variance of the fluxes assigned to each pixel, we used the median average deviation over the data in the pixel and rejected high and low outliers at the $3 \sigma$ level. Pixels with too little data to compute a deviation, i.e., those with $<3$ data points, were also flagged out - these scan-edge pixels are the dominant contribution to the total number of flagged data points. The fraction of the data flagged by all methods was about $0.08 \%$.

\subsection{Creation of the Astrophysical Model}

The timestream data are made into a spatial map using the pointing data corresponding to each time point for each bolometer. The data are weighted by inverse variance across a single scan and then drizzled into a map with 7 .'2 pixels using a nearest-neighbor algorithm. The nearest-neighbor matching allows the map to be returned to a timestream in the same manner, but with the $\mathrm{S} / \mathrm{N}$ improved by averaging over all hits on a given pixel. It has the disadvantage that it accentuates the unevenness of the cross-scan sampling, since each timestream point's value is assigned to an area much smaller than the beam. In principle, this can be addressed by various gridding algorithms and may be a part of future releases of the BGPS data. 

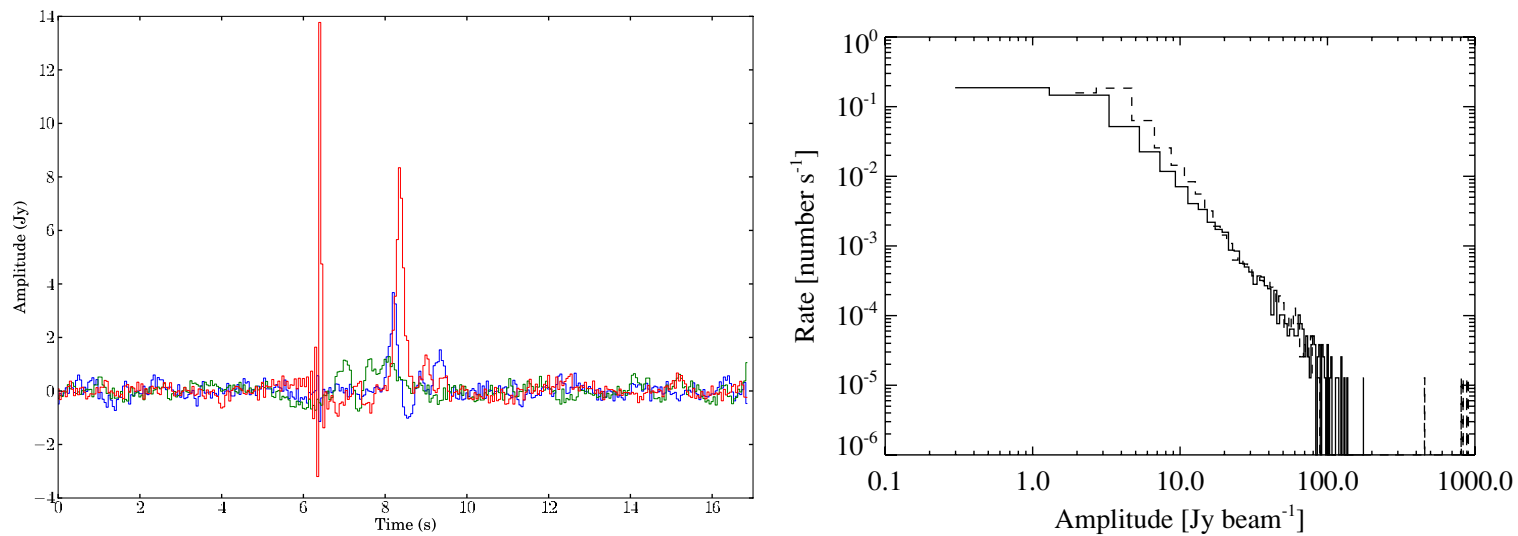

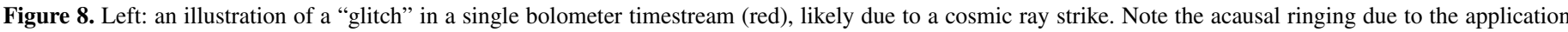

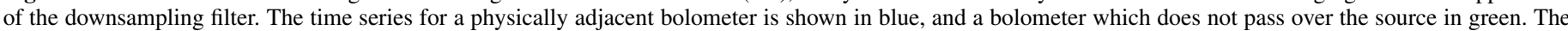

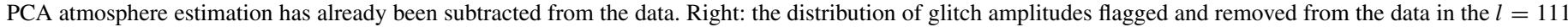

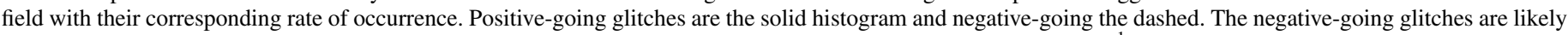

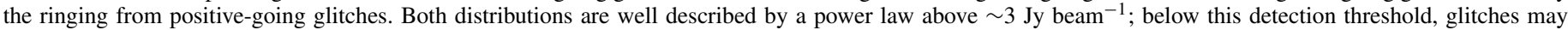
contribute to the overall noise.

(A color version of this figure is available in the online journal.)
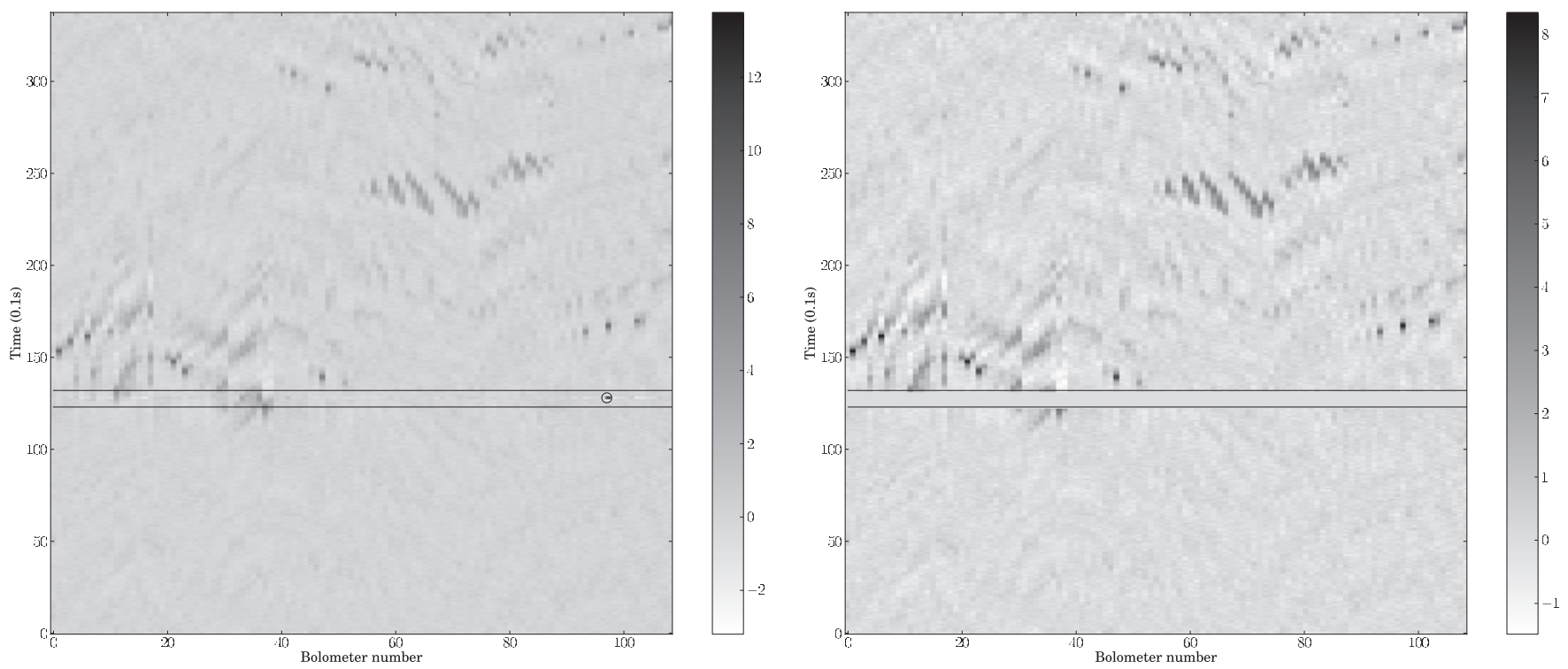

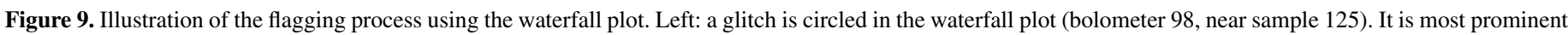

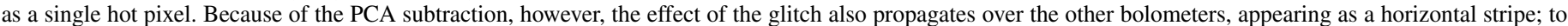

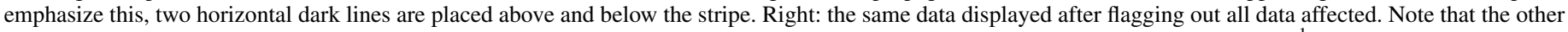
features in the plot correspond to passages of the bolometers over real emission, including NGC 7538 , which has a peak of $\sim 7 \mathrm{Jy}^{\mathrm{beam}}{ }^{-1}$.

The resulting map is then subjected to a maximum-entropy clean algorithm (Hollis et al. 1992) using a specified kernel to produce a deconvolved image which is mapped back into the timestream to be subtracted (Equation (8)). This has several advantages over using the original map directly, including rejecting artifacts smaller than the kernel and decreasing the noise of the resulting timestream. It is also better than a "threshold" method, which produces a model map with sharp discontinuities and also includes noise outliers.

In some fields, flux from some sources spreads out over the course of the iterative process using certain kernel sizes. The cause of this artifact is not yet well understood, but using a different kernel size was an effective workaround and did little to change the properties of the final map. The standard kernel size was a 14".4 FWHM Gaussian; a 21".6 kernel was used when artifacts were encountered. Simulations indicate that this change in kernel has a negligible effect on the flux of the source; see Section 5.1.
We also investigated whether the choice of deconvolution kernel produced a noticeable effect on the recovered flux. Figure 10 demonstrates the effects of using various kernel sizes. The best results, as determined by the flux recovered in simulations and examination of the residual maps, were produced by a kernel smaller than the beam but larger than the pixel size used.

\subsection{Noise Estimation and Residuals}

The weighting of the time stream data used to produce a map is given in Equation (4). If the noise were uncorrelated between timestream points, then $N_{t t^{\prime}}$ would be diagonal. If the noise is correlated between time samples, but is stationary, i.e., the noise properties are time-translation invariant

$$
N_{t t^{\prime}}=N\left(\left|t-t^{\prime}\right|\right)
$$



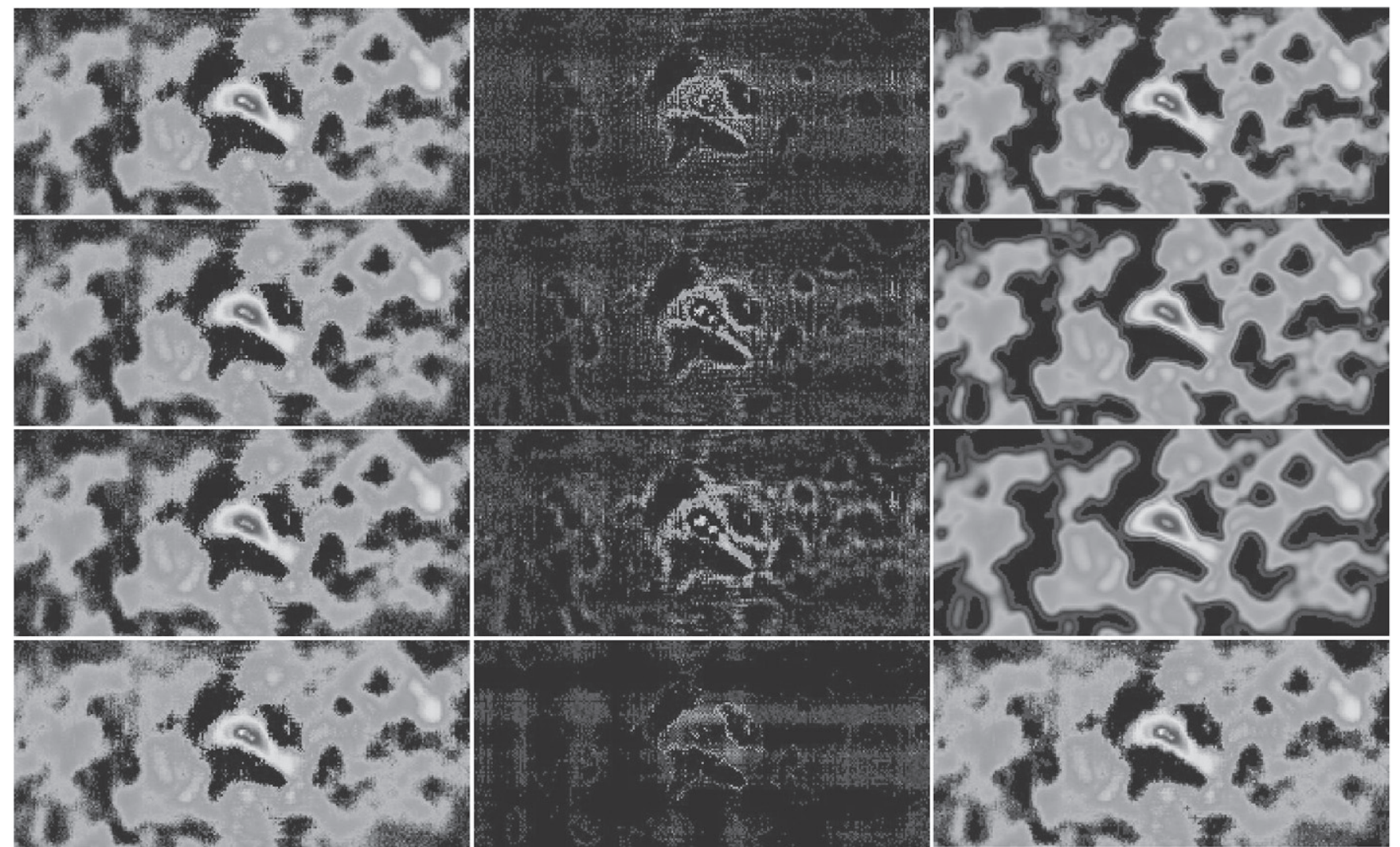

Figure 10. Effect of deconvolution on the iterative process can be seen in its effect on the residuals in these images of Sgr B2, all displayed at the same scale. From left to right: map, residual, model. Top to bottom kernel size: $14^{\prime \prime} 4,21^{\prime \prime}$. $6,31^{\prime \prime} 2,7^{\prime \prime} .2$. The final version of the pipeline uses 14 ."4, which has the result of leaving no flux in the residual at the location of Sgr B2, and does not "dig a hole" in the residual map, as the 7".2 kernel does. The 7".2 kernel also lacks the noise-rejection features of the larger kernels, as can be seen in the bottom right panel.

and the weighting can be done using the Fourier transform of the correlation, which is diagonal:

$$
\tilde{N}_{\omega \omega^{\prime}}=\left\langle|\tilde{\varepsilon}(\omega)|^{2}\right\rangle \delta\left(\omega-\omega^{\prime}\right),
$$

where $|\tilde{\varepsilon}(\omega)|^{2}$ is the power spectrum of the noise $\varepsilon_{t}$. Finally, if the noise is Gaussian, then the covariance matrix provides all the necessary statistical information about the noise.

In practice, few of these assumptions hold for the BGPS data: the noise is not stationary, due to atmosphere variations, and the unsubtracted astrophysical signal causes the residuals from which we estimate the noise to be non-Gaussian. Further, the true remaining correlations between detectors due to unsubtracted atmosphere are difficult to estimate. Our approach, then, is to use a non-optimal but reasonable weighting, estimating the noise from the integral over the power spectrum of the residual timestream for each bolometer and each raster separately. This accounts for the non-stationarity but ignores the correlations, which are small after the PCA subtraction.

The residual maps $E$ (Equation (9)) form the basis for the noise estimation in the map domain and also provide a way of estimating the systematic error resulting from imperfect subtraction of bright sources. These maps are produced for each region from the data residuals after removing the terms $s, a, c, p$, and $e$ in Equation (2). An example of a residual map is shown in Figure 10. In an ideal case, the residual map $E$ represents a map of a realization of the underlying irreducible noise $\varepsilon$. However, the imperfect estimation of the signal results in "ghosts" of bright sources. These features are a guide to the number of iterations necessary and an estimate of the remaining systematic error. In constructing the BGPS catalog, R10 found that smoothed versions of the residual maps provide a reliable means of determining the pixel-to-pixel error which accounts for the local variations in the noise, including those due to such artifacts (cf. R10, Figure 2). Because observing conditions varied widely during the survey, the rms noise level varies over the BGPS. However, the noise within a given $1^{\circ} \times 1^{\circ}$ field is fairly uniform in the absence of very bright sources. The noise level varies between 11 and $53 \mathrm{mJy}_{\text {beam }}^{-1}$ in the inner Galaxy. We show the variation of the depth as a function of Galactic longitude in Figure 11. This noise level interacts with the source density to produce the completeness of source extraction. The variation of completeness as a function of Galactic longitude, determined by simulations of the source extraction, is shown in R10's Figure 9.

\section{CALIBRATION AND PHOTOMETRY}

\subsection{Flux Density Calibration}

The absolute flux calibration is derived from observations of Mars and Uranus (the "primary calibrators"). The millimeterwave flux of these planets is known to 5\% (Orton et al. 1986; Griffin \& Orton 1993). The fluxes from these models were extracted using the JCMT's FLUXES ${ }^{15}$ program with a central frequency $271.1 \mathrm{GHz}$ and bandwidth $46.0 \mathrm{GHz}$ (Table 4).

Calibrations at (sub)millimeter wavelengths are strongly affected by atmospheric opacity corrections. Further, the detector

\footnotetext{
15 http://docs.jach.hawaii.edu/star/sun213.htx/sun213.html
} 


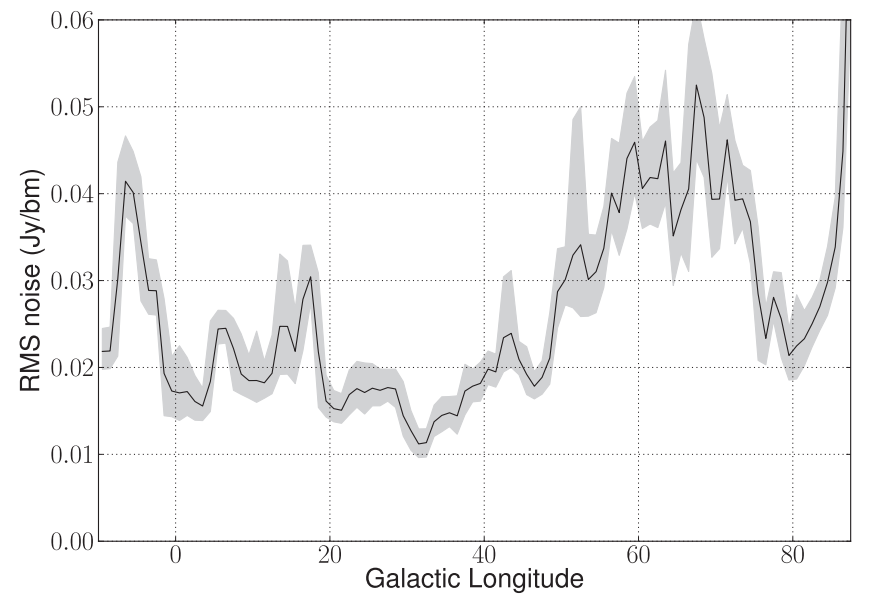

Figure 11. Variation of rms noise as a function of Galactic longitude for the fields in the inner Galaxy. The noise reaches a minimum at $l=31$ at $11 \mathrm{mJy}^{-1}$ beam ${ }^{-1}$ and a maximum at $l=57$ at $57 \mathrm{mJy} \mathrm{beam}^{-1}$. The shaded region shows the range within which $68 \%$ of the rms measurements in a field lie.

responsivity of Bolocam's bolometers is a nonlinear function of the mean atmospheric loading. To address both of these problems and relate observations of the primary calibrators to observations of the BGPS fields, we make use of the following relation. The calibration $\mathcal{C}$, referenced to the detectors, is given by

$$
\mathcal{C}\left[\frac{\mathrm{V}}{\mathrm{Jy}}\right]=\mathcal{R}(\tau) \eta A \exp (-\tau) \Delta \nu,
$$

where $\mathcal{R}$ is the bolometer responsivity $\left(\mathrm{V} \mathrm{W}^{-1}\right), \eta$ is the system optical efficiency, $A$ is the effective telescope collecting area, $\Delta v$ is the bandwidth, and $\tau$ is the line-of-sight, inband atmosphere opacity. Under the assumption that the only power variation on the detectors is due to the power from the atmosphere (i.e., that astronomical sources are faint relative to the atmosphere), which may be parameterized by $\tau, \mathcal{C}$ is a single-valued function of $\tau$. We have used the measured potential difference across the detector thermistor $V_{\mathrm{DC}}$ as a proxy for $\tau$, since the bolometer resistance is a single-valued function of loading. This quantity is monitored continuously for all observations. Note that this calibration curve folds in both the effects of changing atmospheric transmission and the changes in the detector response with optical loading.

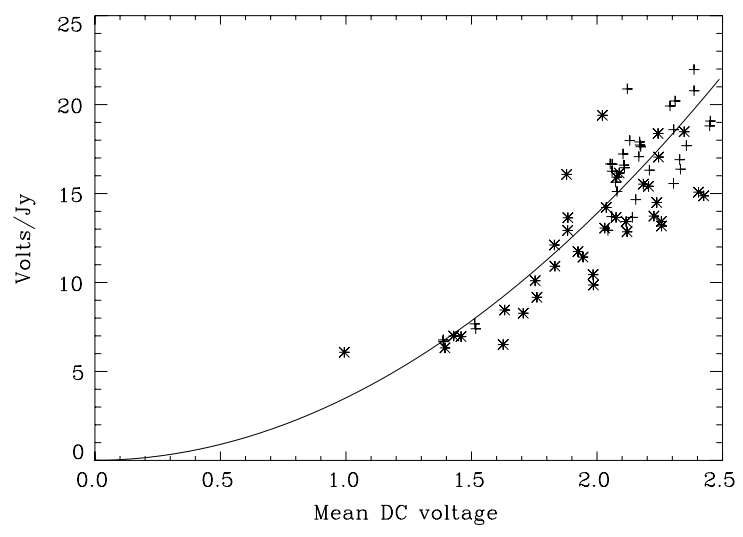

The calibrator field observations were obtained and reduced differently from the science fields. Calibrators were observed in $\sim 8^{\prime}$ radius fields with two sets of observations, scanned in orthogonal directions. In these small fields, the iterative process tended to diverge with more iterations because of instabilities in the deconvolution procedure. Therefore, the calibration curve was determined from non-iteratively-mapped calibrator maps. A future work will explore applying a more consistent application of the processing to both calibrator and science fields.

A fit to the observed values $\left(V_{\mathrm{DC}}\right.$ versus $\mathcal{C}$ ) for the primary calibrators was performed for each epoch separately. The agreement between epochs was good, so a single combined calibration was used for all data. The calibration curve is shown in Figure 12. The resulting error on the calibration curve fit is less than $8 \%$ (statistical) over the observed range of $\tau$.

The above flux density calibration only accounts for the average calibration of bolometer Volts to Janskys. It is also necessary to account for the variation of bolometer response across the focal plane (often referred to as "flat-fielding"). We do this by monitoring the response to the atmosphere emission in all bolometers (astrophysical signal is negligible in comparison to the sky except toward SgrB2). Being in the near field, the atmosphere is common to all detectors, and thus this serves as a common relative reference to calibrate out variation in the individual detector responsivities and optical efficiencies. (For more detail regarding the properties of atmospheric noise above Mauna Kea as inferred from the Bolocam data, see Sayers et al. (2010).) The change in relative response with loading for a typical detector is shown in Figure 12. The actual application of the method to the time series of the bolometer data is shown in Figure 13, where it is clear that correlation of the timestreams is indeed improved by the application of a single multiplicative factor (the $r_{i}$ of Equation (12)).

\subsection{Point-spread Function, Aperture Corrections, and Surface Brightness Calibration}

The Bolocam point-spread function (PSF, or "beam") is measured using the planets Uranus and Neptune, which are nearly point sources for Bolocam. By stacking all observations of planets, we obtain a high $\mathrm{S} / \mathrm{N}$ profile of the beam; this is shown in Figure 14. The main lobe is fit to a Gaussian profile. This yields the effective beam FWHM of 33", corresponding

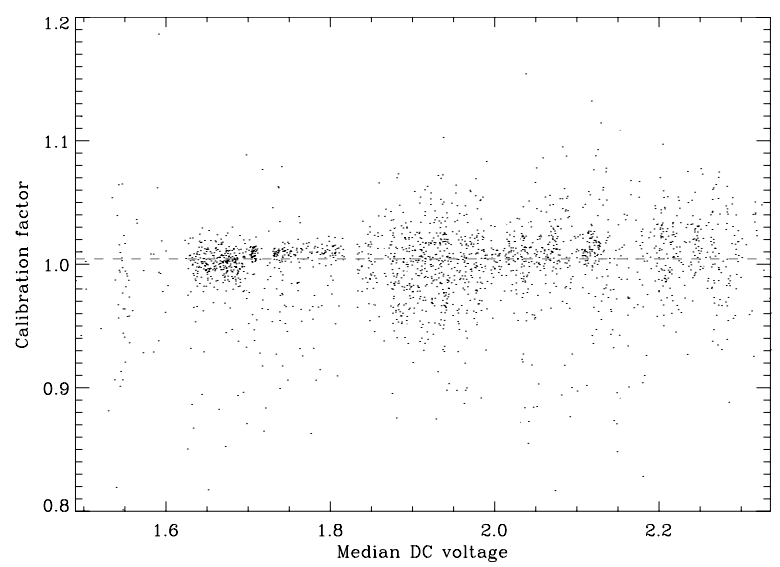

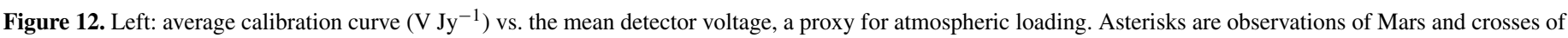

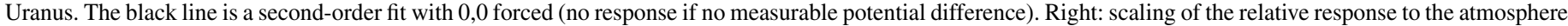

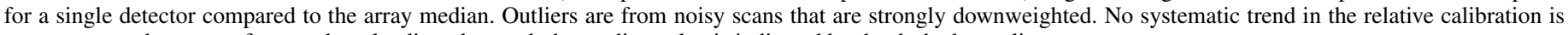
apparent over the range of atmosphere loading observed; the median value is indicated by the dashed gray line. 


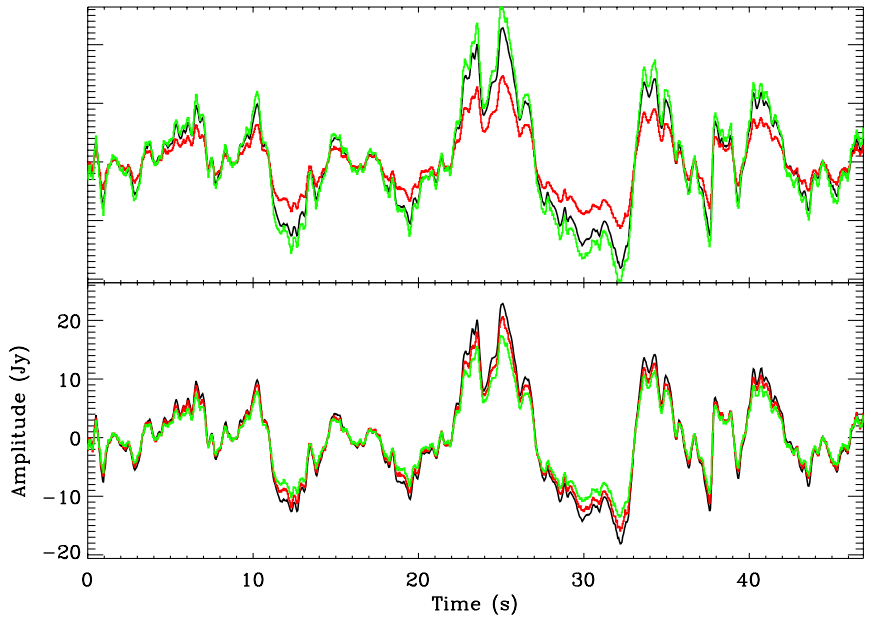

Figure 13. Illustration of the relative sensitivity calibration using the atmosphere as a calibrator. Black is the median over all bolometers (the first-order atmosphere model), red and green are individual bolometers before (top) and after (bottom) applying the relative calibration. Note the improved agreement.

(A color version of this figure is available in the online journal.)

to a sigma of $14^{\prime \prime} .2$ and equivalent top-hat radius of 19.8 . The corresponding solid angle is $2.9 \times 10^{-8} \mathrm{sr}$.

The calibration from Jy beam ${ }^{-1}$ into surface brightness (MJy $\mathrm{sr}^{-1}$ ) is obtained once the beam area is determined. For the BGPS, the conversion factor is

$$
1 \mathrm{Jy}_{\text {beam }}{ }^{-1}=34.5 \mathrm{MJy} \mathrm{ster}^{-1}=0.042 \mathrm{Jy} \mathrm{pixel}^{-1}
$$

for the standard $7^{\prime \prime} .2$ pixel size. The uncertainty in the mean beam diameter is $3 \%$, leading to a corresponding $6 \%$ uncertainty in the beam area (and in the surface brightness calibration). This factor is in addition to the uncertainty in the best-fit $V_{\mathrm{DC}}$ versus $\mathcal{C}$ curve.

Due to the sidelobes of the beam, aperture photometry may not capture all of the flux from a point source if the aperture is too small. R10 used a standard set of apertures, and the multiplicative correction factors to be applied to fluxes obtained from these apertures for a point source are given in Table 2. The correction is only significant for apertures $\leqslant 80^{\prime \prime}$, beyond which effectively all of the flux is included. For extended objects,
Table 2

Point Source Aperture Corrections

\begin{tabular}{lc}
\hline \hline R10 Aperture & Recommended Correction \\
\hline $40^{\prime \prime}$ & 1.46 \\
$80^{\prime \prime}$ & 1.04 \\
$120^{\prime \prime}$ & 1.00 \\
Object integrated & 1.00
\end{tabular}

the situation is more complicated because of the effects of the mapping algorithm. This is discussed further in the next section.

\subsection{Effects of the Data Reduction Procedures on Photometry}

To do accurate and meaningful photometry on the Bolocam maps requires a good estimate of the noise (Section 4.6) but also an understanding of the spatial filtering imposed by the observing strategy and the cleaning and mapping of the data. The additional complexities of defining "sources" for the complicated morphologies actually observed are discussed in Rosolowsky et al. (2010). Here, we restrict our discussion to the limitations on performing photometry imposed by the data reduction.

The fundamental feature of the data is that there is a degeneracy between large spatial scale astrophysical emission and the atmosphere and other signals with long spatial or temporal variations. Consequently, there are limits to the ability of the algorithm to separate the signal $s$ from the atmosphere $a$ and time-correlated terms $c$ in Equation (6). Inevitably, some of the signal which should be present in $s$ is mixed into these terms and is thus not present in the final map $m$. Because $s$ is generated from a deconvolution algorithm, it has non-zero mean by construction, but importantly, it does not contain an unbiased estimate of the astrophysical signal on all spatial scales: large spatial scales are preferentially attenuated.

The use of a constant factor to convert to surface brightness implicitly assumes that the response to all angular scales larger than the beam is the same. In fact, the data reduction process acts differently on sources of different angular extent. In performing photometry, the flux density in an aperture should in fact be written as

$$
F=f\left(\Omega_{\text {src }}\right) \int_{\text {aper }} S / \Omega_{b} d \Omega,
$$
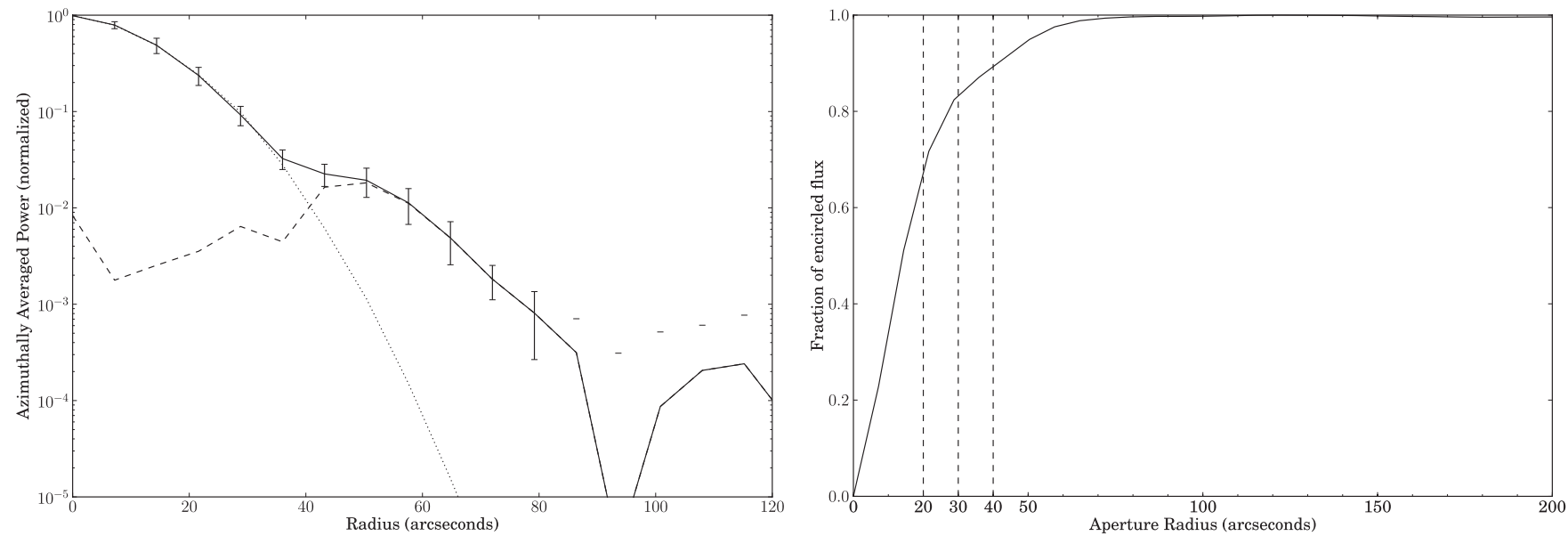

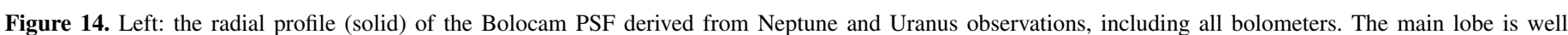

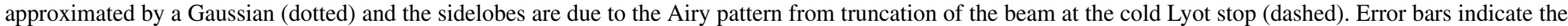

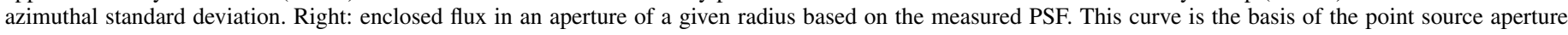
corrections (Table 2). Vertical dashed lines indicated 20,30, and 40 arcsec radii. 


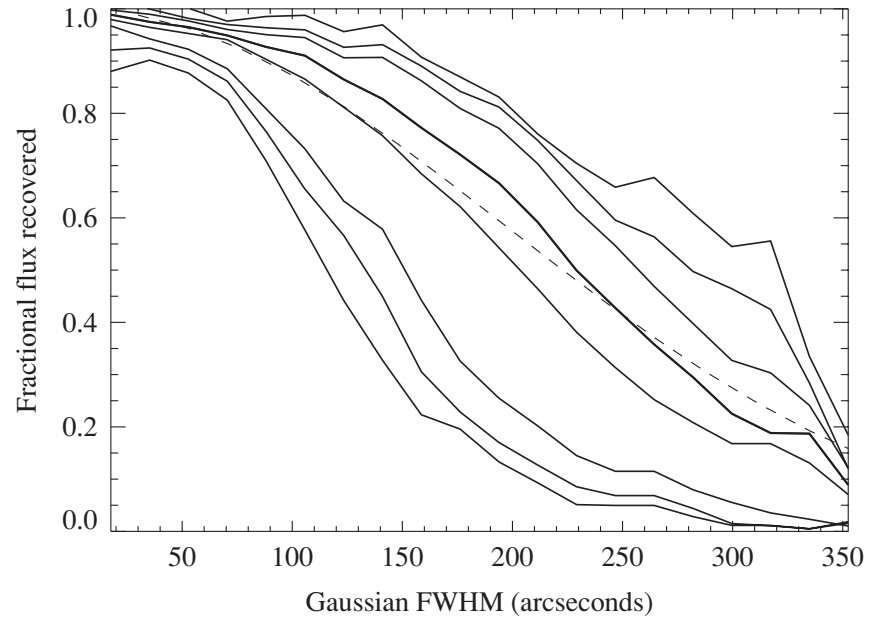

Figure 15. Fractional flux recovered as a function of source size for wellseparated Gaussian sources with FWHM as indicated. The thin lines show (from top to bottom) the effect of increasing the number of PCA components subtracted $(3,7,10,13,16,21,26$, and 31$)$. The thick line shows the curve for the 13 PCA component cleaning used in the released data; the flux recovery drops to $50 \%$ at 3!.8 for this case. The three and seven PCA cases show bumps because atmospheric noise is still present at large scales. The dashed line shows the approximation of a linear high pass filter with "brick wall" cutoff below $1 / 10$ inverse arcminutes.

where $S$ is the flux density in each pixel in $\mathrm{Jy}_{\text {beam }}{ }^{-1}, \Omega_{b}$ is the beam solid angle in steradians, and $f\left(\Omega_{\mathrm{src}}\right)$ is a correction factor depending on the true angular size of the source. The factor $f$ can deviate significantly from unity for extended sources, and as $\Omega_{\text {src }}$ is generally unknown, this leads to the largest systematic uncertainty in obtaining photometric fluxes.

To determine the factor $f\left(\Omega_{\text {src }}\right)$ (the flux recovered as a function of angular scale of the source), we performed a series of simulations. Gaussian sources with a range of peak flux densities and sizes were inserted on top of a background consisting of real BGPS time series data with the astrophysical source model removed. The simulated time series were then processed identically to the real data. The recovered fluxes were measured in ellipses that included single well-separated sources. Figure 15 shows the fraction of flux recovered in the map as a function of source size for a range of different PCA components subtracted in the cleaning. The final reduction used 13 PCA components because this produced a reasonable compromise between attenuating extended structure and cleaning the atmospheric contribution. Note that this is a substantially more aggressive cleaning than that used in the reduction of the Bolocam observation of the Spitzer cores-to-disks fields (Enoch et al. 2006, 2007; Young et al. 2006), largely because our mean integration time per pixel was $\sim$ seconds, whereas theirs was $\sim$ minutes. The recovered flux does depend on the source size, but importantly does not depend on the source strength except for the strongest sources (e.g., SgrB2).

While the precise effect of the flux reduction requires a full simulation of the data reduction pipeline to ascertain, the flux reduction effect can be reproduced with a relatively simple prescription which is intuitively motivated. Because the atmosphere model $a$ and PCA model $c$ remove from the timestream "signals" which are correlated spatially across the array at the same time, this effectively acts as a spatial filter on the map, removing spatial scales comparable to the array FOV. We have found that a simple "brick wall" filter in Fourier space which nulls all modes below 0.1 inverse arcminutes (spatial

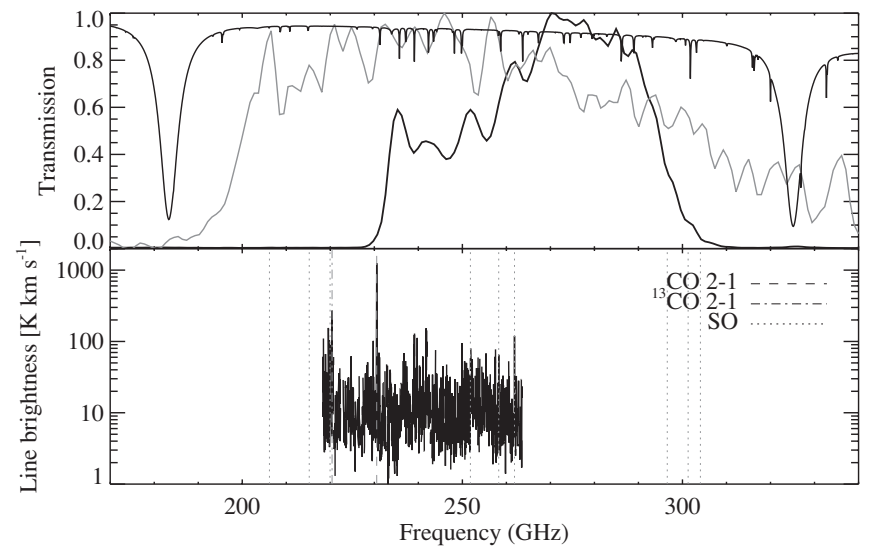

Figure 16. Top: the Bolocam $1.1 \mathrm{~mm}$ bandpass (thick line). Also shown is the atmospheric transmission at Mauna Kea for $1 \mathrm{~mm}$ of precipitable water vapor (thin line). The gray curve is the MAMBO-2 bandpass (taken from http://www.mpifr-bonn.mpg.de/div/bolometer/mambo_parameters 020430.html). Note that the Bolocam bandpass is well away from the strong water absorption features at 183 and $325 \mathrm{GHz}$. Bottom: the effect of line contamination is illustrated with the integrated line intensities from the Nummelin et al. (1998) survey of Sgr B2, who found that $22 \%$ of the flux density in one pointing was due to line emission. Note that the Bolocam passband rejects $>90 \%$ of the ${ }^{12} \mathrm{CO}$ flux. However, as shown from the Nummelin et al. (1998) survey, $\mathrm{SO}_{2}$ and $\mathrm{CH}_{3} \mathrm{OH}$ lines can be strong contributors to line flux in the passband due to their broad width. Other lines lying in the Bolocam passband include $\mathrm{CS}(5 \rightarrow 4)$ and $(6 \rightarrow 5)(245$ and $293 \mathrm{GHz}), \mathrm{HCN}(3 \rightarrow 2)(265 \mathrm{GHz})$, and $\mathrm{HCO}^{+}(3 \rightarrow 2)(267 \mathrm{GHz})$.

scales larger than $10^{\prime}$ ) reproduces very well the attenuation seen in the simulation. The comparison between the flux attention produced by this filter and that found from simulation is shown in Figure 15. The implication is that the BGPS is not sensitive at all to scales larger than $10^{\prime}$.

\subsection{Bandpass Effects: Color Corrections and Line Contamination}

The width of the Bolocam passband is such that the effective band center, or equivalently, the flux density referred to a fixed band center, will change somewhat depending on the source SED. Atmospheric transmission variations may also affect the effective passband. Figure 16 shows the passband. Bolocam is largely insensitive to variations in the strong water absorption lines at 183 and $325 \mathrm{GHz}$. We compute color corrections due to changes in the source spectrum in Appendix A.

Note that we have made no correction for the contamination of the continuum flux densities by emission from lines. The Bolocam passband was specifically constructed to exclude the strong ${ }^{12} \mathrm{CO}(2 \rightarrow 1)$ emission line at $230 \mathrm{GHz}$. We can estimate the fractional contribution of a line to the continuum as

$$
\begin{aligned}
\frac{L}{C} & =\frac{J \int T \phi(v) t(v) d v}{\int S_{v} t(v) d v} \approx \frac{J T \Delta v v_{c}}{S_{v} \Delta v c} \\
& =0.01\left(\frac{T \Delta v}{10 \mathrm{~K} \mathrm{~km} \mathrm{~s}^{-1}}\right)\left(\frac{1 \mathrm{Jy}}{S_{v}}\right)
\end{aligned}
$$

where $T$ is the line strength in Kelvin, $\phi(v)$ is the line shape (of width $\Delta v$ in $\mathrm{km} \mathrm{s}^{-1}$ ), $t(v)$ is the Bolocam passband (with equivalent bandwidth $\Delta v$ and bandcenter $v_{c}$ ), and $J$ is given by Equation (1). The approximation holds for lines in the center of the band. The scaling values are chosen to be typical of the line-to-continuum actually seen. Thus, any given line is likely to at most contribute a few percent to the measured flux, but in the most extreme star-forming environments (e.g., 

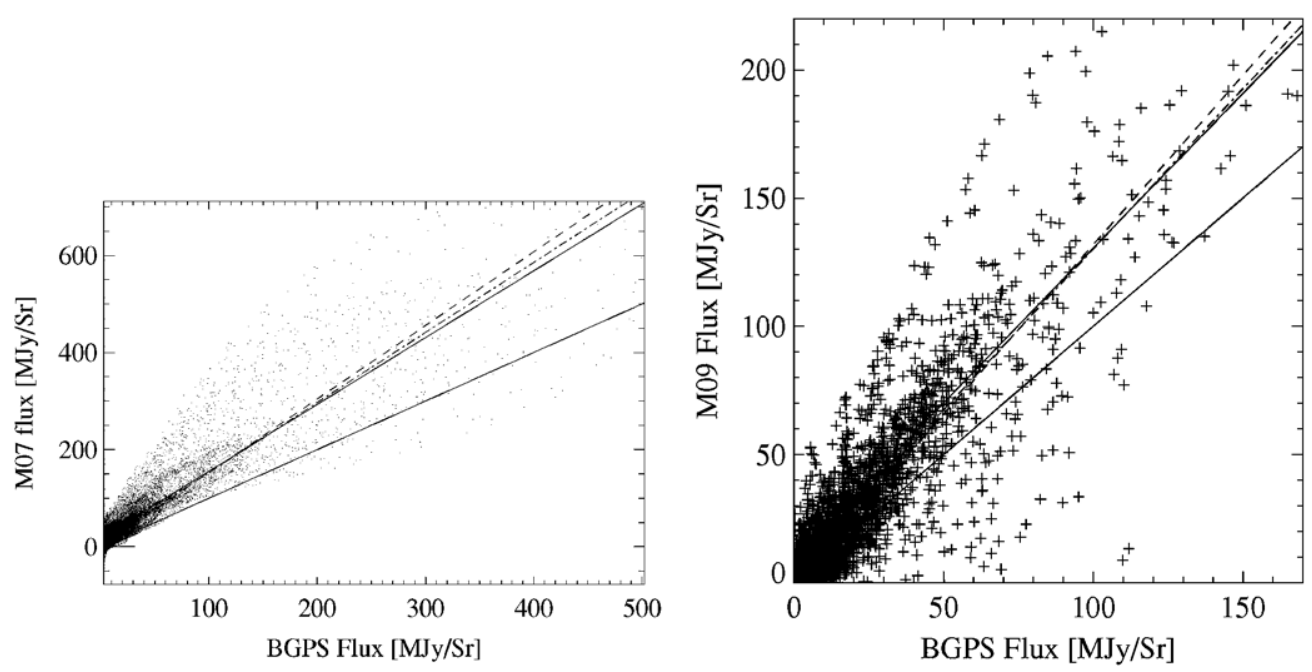

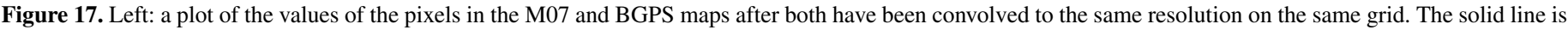

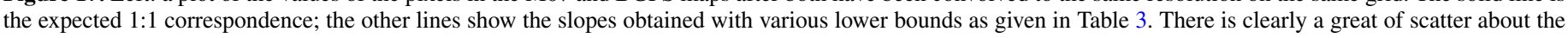
mean. Right: the same analysis, but for the M09 SIMBA data.

Sgr B2) we may expect that lines may contribute a substantial fraction of the total measured flux density due to the integrated effect of the lines. For an example, see the lower panel of Figure 16.

For completeness, we note that toward $\mathrm{H}$ II regions, free-free emission may represent a non-trivial fraction of the millimeter flux density in addition to the thermal dust continuum. This effect is of course best assessed on a case-by-case basis by comparing the Bolocam maps with radio surveys, e.g., the VGPS (Stil et al. 2006).

\subsection{Comparison to Other Surveys}

Comparison of the Bolocam flux densities is easiest for an instrument with the same or similar bandpass, since the additional complication of the source spectral index is avoided. For this reason, we choose to make an initial comparison of the accuracy of our calibration with the MAMBO and SIMBA instruments, since their passbands at $1.2 \mathrm{~mm}$ are quite close to that of Bolocam. MAMBO operates on the IRAM $30 \mathrm{~m}$ diameter telescope on Pico Veleta in Spain, and SIMBA operated on the $15 \mathrm{~m}$ SEST antenna prior to that facility's closure. An important future cross-check will be comparison of the BGPS flux density calibration with SCUBA $850 \mu \mathrm{m}$ or ATLASGAL $870 \mu \mathrm{m}$ results.

The study of the Cygnus- $X$ region by Motte et al. (2007) (hereafter M07) presents one of the largest fields in the Galactic plane that has been mapped with MAMBO which overlaps with the Bolocam survey fields. M07 used both the 37 element MAMBO and 117 channel MAMBO-2 instruments. The FWHM beam size for the observations was $11^{\prime \prime}$. The MAMBO beam model resulting from the chopping-scanning observing strategy is described in the Appendix of Motte \& André (2001). A detailed description of the MAMBO data reduction is given in Kauffmann et al. (2008). M07 used Uranus and Mars as their fundamental flux calibration, with an estimated uncertainty in the flux of $20 \%$. The effective passband of the images presented by M07 is about $90 \mathrm{GHz}$ centered at $260 \mathrm{GHz}$ and thus includes the bright $J=2-1 \mathrm{CO}$ transition (see Figure 16).

A survey of $2 \operatorname{deg}^{2}$ toward $\ell=44^{\circ}$ was conducted by Matthews et al. (2009, hereafter M09). These images have 22"resolution, and unlike the M07 survey, which used chopping and scanning simultaneously, the SIMBA data were obtained in fast scan mode only.

To compare the BGPS images to the M07 and M09 images, we first obtained the data in FITS image format. The M07 data are publicly available ${ }^{16}$. The M09 data were kindly provided by H. Kirk (2010, private communication). All data were regridded to a common projection and coordinate system grid with $4^{\prime \prime}$ pixels using the program Montage ${ }^{17}$. We then converted to $\mathrm{MJy} \mathrm{sr}^{-1}$ in a given pixel based on the beam and pixel sizes. Finally, the Bolocam map was convolved with either an $11^{\prime \prime}$ (M07) or 22"(M09) FWHM Gaussian and the M07 and M09 maps are convolved with a $33^{\prime \prime}$ FWHM Gaussian so that the effective resolution of the two pairs (BGPS-M07, BGPS-M09) is the same. The convolution preserves the total flux in the map, but changes the aperture in which that flux appears. This results in maps which, were the calibration and observed features in each map identical, can be compared on a pixel-by-pixel basis.

The plots of pixel values versus each other are shown in Figure 17. The BGPS values are indeed correlated with both M07 and M09 over a range of about two orders of magnitude, but with a large amount of scatter. Some of the scatter is due to noise, but the larger effect is likely the difference in the handling of extended structure between the different surveys. However, the average value of the correlation coefficient deviates significantly from 1 in comparing to both M07 and M09, with a multiplicative factor of 1.21-1.51 required to make the BGPS fluxes match the other surveys (or $0.66-0.83$ to make the other surveys agree with the BGPS). This factor also depends on the range over which the fit is done, as given in Table 3. Including all pixels down to $3 \mathrm{MJy} \mathrm{sr}^{-1}$ produces systematically larger required correction factor. Using the average value (to account for variation of the factor with brightness) yields a factor of 1.35 . For a dust spectral index of $\sim 3.5$, the $1.2 \mathrm{~mm}$ measurements should have produced

\footnotetext{
$16 \mathrm{ftp} / / / \mathrm{cdsarc} . \mathrm{u}-\mathrm{strasbg.fr} / \mathrm{pub} / \mathrm{cats} / \mathrm{J} / \mathrm{A}+\mathrm{A} / 476 / 1243 / \mathrm{fits} /$

17 This research made use of Montage, funded by the National Aeronautics and Space Administration's Earth Science Technology Office, Computation Technologies Project, under Cooperative Agreement Number NCC5-626 between NASA and the California Institute of Technology. Montage is maintained by the NASA/IPAC Infrared Science Archive.
} 
Table 3

Flux Comparison with M07 and M09

\begin{tabular}{cccc}
\hline \hline $\begin{array}{c}\text { Comparison } \\
\text { Survey }\end{array}$ & $\begin{array}{c}\text { Factor } \\
\mathrm{MJy} \mathrm{sr}^{-1}\end{array}$ & $\begin{array}{c}\text { Factor } \\
>10 \mathrm{MJy} \mathrm{sr}^{-1}\end{array}$ & $\begin{array}{c}\text { Factor } \\
>20 \mathrm{MJy} \mathrm{sr}^{-1}\end{array}$ \\
\hline M07 & 1.32 & 1.25 & 1.21 \\
M09 & 1.51 & 1.44 & 1.38 \\
\hline
\end{tabular}

Table 4

Comparison of Band Centers, Color Corrections, and Flux Ratios for Bolocam and MAMBO

\begin{tabular}{ccccc}
\hline \hline$\alpha^{\mathrm{a}}$ & $\begin{array}{c}\text { Bolocam } \\
v_{c}(\mathrm{GHz})\end{array}$ & $\begin{array}{c}K \\
\text { Equation }(\mathrm{A} 5)\end{array}$ & $\begin{array}{c}\text { MAMBO } \\
v_{c}(\mathrm{GHz})\end{array}$ & $S_{\text {BOLOCAM }} / S_{\text {MAMBO }}$ \\
\hline$\overline{-}^{\mathrm{c}}$ & 271.1 & 1.000 & 262.0 & 1.124 \\
\hline 1.0 & 267.8 & 0.988 & 254.8 & 1.051 \\
1.5 & 268.6 & 0.986 & 256.4 & 1.072 \\
2.0 & 269.4 & 0.987 & 258.0 & 1.090 \\
2.5 & 270.0 & 0.990 & 259.5 & 1.104 \\
3.0 & 270.7 & 0.996 & 261.1 & 1.115 \\
3.5 & 271.4 & 1.004 & 262.5 & 1.124 \\
4.0 & 272.2 & 1.015 & 264.0 & 1.130 \\
4.5 & 272.9 & 1.030 & 265.4 & 1.137 \\
5.0 & 273.8 & 1.049 & 266.8 & \\
\hline
\end{tabular}

Notes.

a The spectral index as defined by Equation (A4).

b The expected ratio of flux densities, assuming identical apertures, the passbands of Figure 16, and the SED indicated.

${ }^{c}$ The fiducial SED is taken to be that of Equation (A3) with $T=20 \mathrm{~K}, \beta=1.8$, and unity optical depth at $100 \mu \mathrm{m}$.

a flux that is about $12 \%$ lower than that measured by BGPS (see Table 4). Assuming this is a typical spectral index for these clumps, we therefore note that one must multiply by a factor of $1.35 \times 1.12=1.5$ (with a range of plus or minus 0.15 ) the Version 1.0 images and catalog released through IPAC to obtain good agreement with these surveys.

The source of this discrepancy remains unclear. It is unlikely to be due to line contamination in the MAMBO/SIMBA bands. Line contamination could increase the continuum flux by at most a few percent for MAMBO (see Equation (18)), while being negligible for Bolocam. For most sources, it is also not obvious that the discrepancy is due to filtering of extended structure. The filtering does not reach the requisite $50 \%$ needed to explain the difference until the source FWHM reaches 230" (Figure 15). However, the simulations were run on Gaussian sources, and it is still not certain whether the filter function differs for other source shapes. We do not understand the effective filter functions of the SIMBA and MAMBO surveys well enough to include their modeling in the comparison.

Work is ongoing to thoroughly investigate all potential sources of this discrepancy with further more realistic simulations, a new treatment of atmosphere removal, and application of distortion corrections. In addition, the processing for bright flux density calibrators in the current pipeline is different from the processing of the science fields, and attempts are being made to process the different observation types with an identical pipeline. This analysis will be discussed in a future work.

\section{FINAL MAPS AND DATA RELEASE}

The final maps are produced by co-adding all observations of a given region. The maps were processed in 29 separate pieces. The maps are made in Galactic coordinates using a plate carré
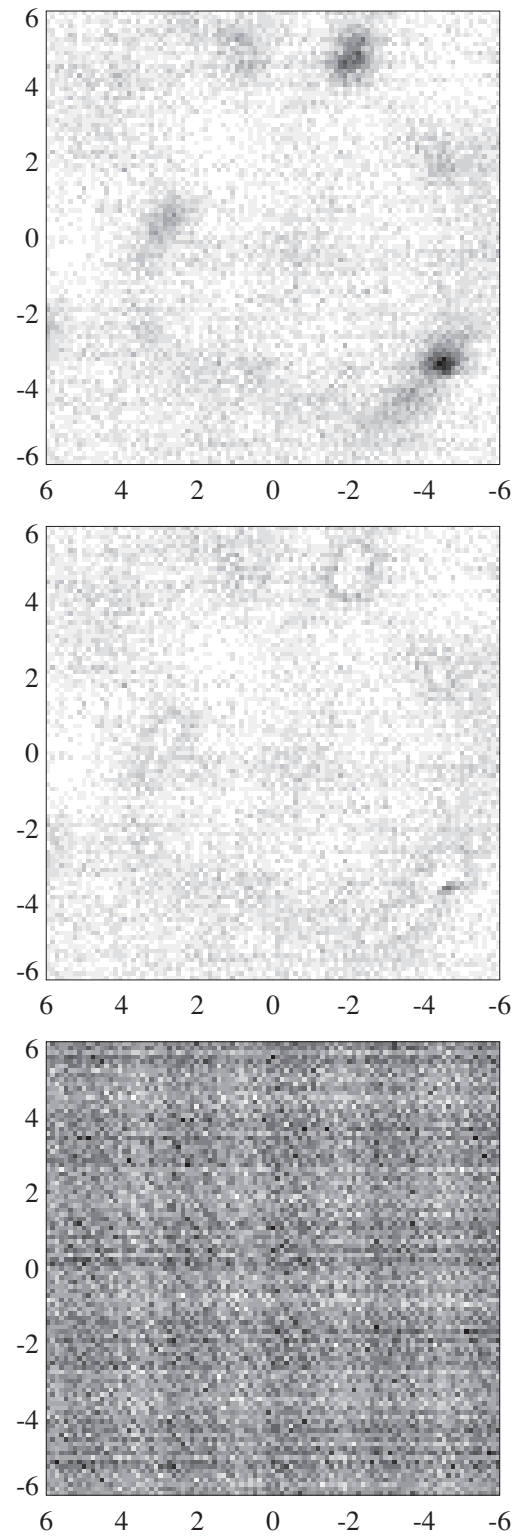

Figure 18. Examples of the three image types in the IPAC V1.0 data release. Images are zoomed in to show detail; axes are offsets in arcminutes from the center position. Top left: MAP. Middle: NOISEMAP. Bottom: NHITSMAP. Note the slight "ghosts" in the residual (NOISEMAP) at the positions of bright sources. Note also the "basket-weave" pattern in the coverage (NHITSMAP) due to gaps in the detector array combined with the scan strategy.

(FITS header CAR) projection. (This is the same projection used by the Spitzer-GLIMPSE and BU-FCRAO GRS surveys.) By placing them all on the same equatorial grid, mosaicking the maps together is straightforward. As these maps are near the coordinate system equator, the difference between truly equiareal pixels and the pixels used is at most $0.4 \%$, even $5^{\circ}$ out of the plane (as for IC1396). The pixel size is 7".2, chosen to be much smaller than the Bolocam beam. The maps are written to standard FITS files. An example of the FITS header for the BGPS images is given in Appendix B.

All processed maps are available through IPAC $^{18}$. IPAC provides a cutout service for the images as well as a searchable version of the catalog provided in Rosolowsky et al. (2010).

\footnotetext{
$\overline{18 \text { http://irsa.ipac.caltech.edu/Missions/bolocam.html }}$
} 

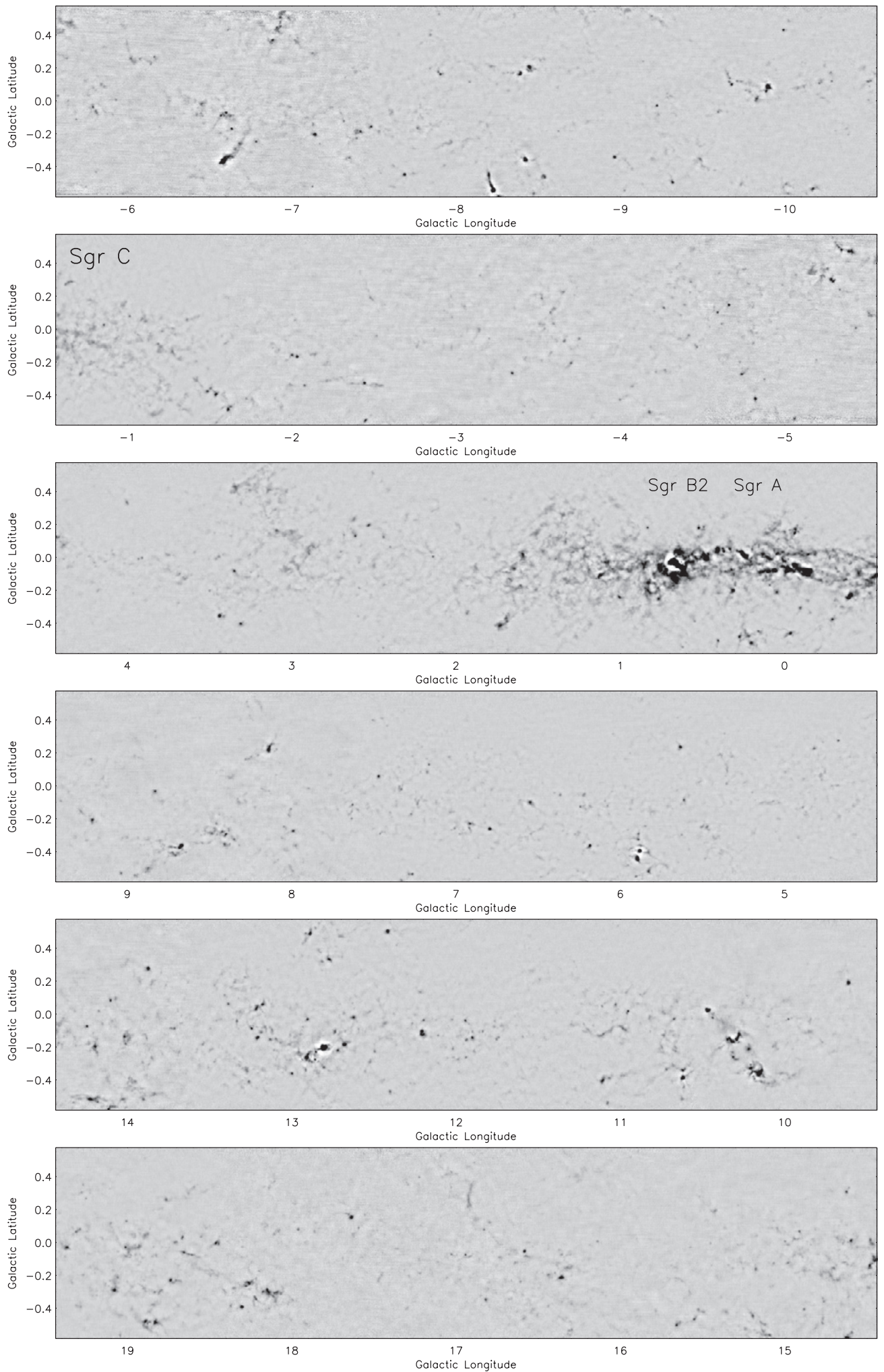

(a)

Figure 19. Images from the BGPS. (a) $l=-10.5$ to $l=19.5$. In this figure and the following, the brightest sources, e.g., Sgr B2, Sgr A, and sources near $l=10$ and $l=13$, appear to be saturated, but this is only a display artifact. The astrophysical sources are always much fainter than the atmosphere (which is within the dynamic range of the detectors) and therefore do not saturate. The noise is more pronounced from $l=-7$ to $l=-2$ because this region was observed less. (b) $l=19.5$ to $l=49.5$. G34.3+0.15, W 51, W 43, W 49, and M 17 appear to be saturated, but this is only a display artifact. The $20<\ell<40$ region through the 4-8 kpc molecular ring and approximately the termination of the galactic bar is particularly rich in clumps. (c) $l=49.5$ to $l=74.5$. In comparison to the inner galaxy, the $65<\ell<75$ has a very sparse population of faint clumps. (d) Top: the Cygnus Arm. Note that coverage in $b$ is extended to $\pm 1.5 \mathrm{deg}$. Bottom: a zoom-in of the region near DR21, showing at left the data from the M07 IRAM study of this region, and at right the BGPS map, in which the filamentary nature of the emission is more apparent. Flux densities from the BGPS and M07 are compared in Section 5.5. (e) The IC1396 region. In spite of copious CO emission here, there are only two faint sources detected in $9 \mathrm{deg}^{2}$. (f) Cloud complexes centered at $l=111$ in the Perseus Arm. The NGC 7538 complex is in the upper left. (g) W3. The W3(OH)/W3 Main complex is the bright source on the right side of the image. (i) W4/5. W5 has one scan performed in R.A./decl. instead of Galactic coordinates and so has non-uniform noise properties in each square degree. (j) Gem OB1. This region has been thoroughly surveyed in $\mathrm{NH}_{3}$ and is discussed in Dunham et al. (2010). 

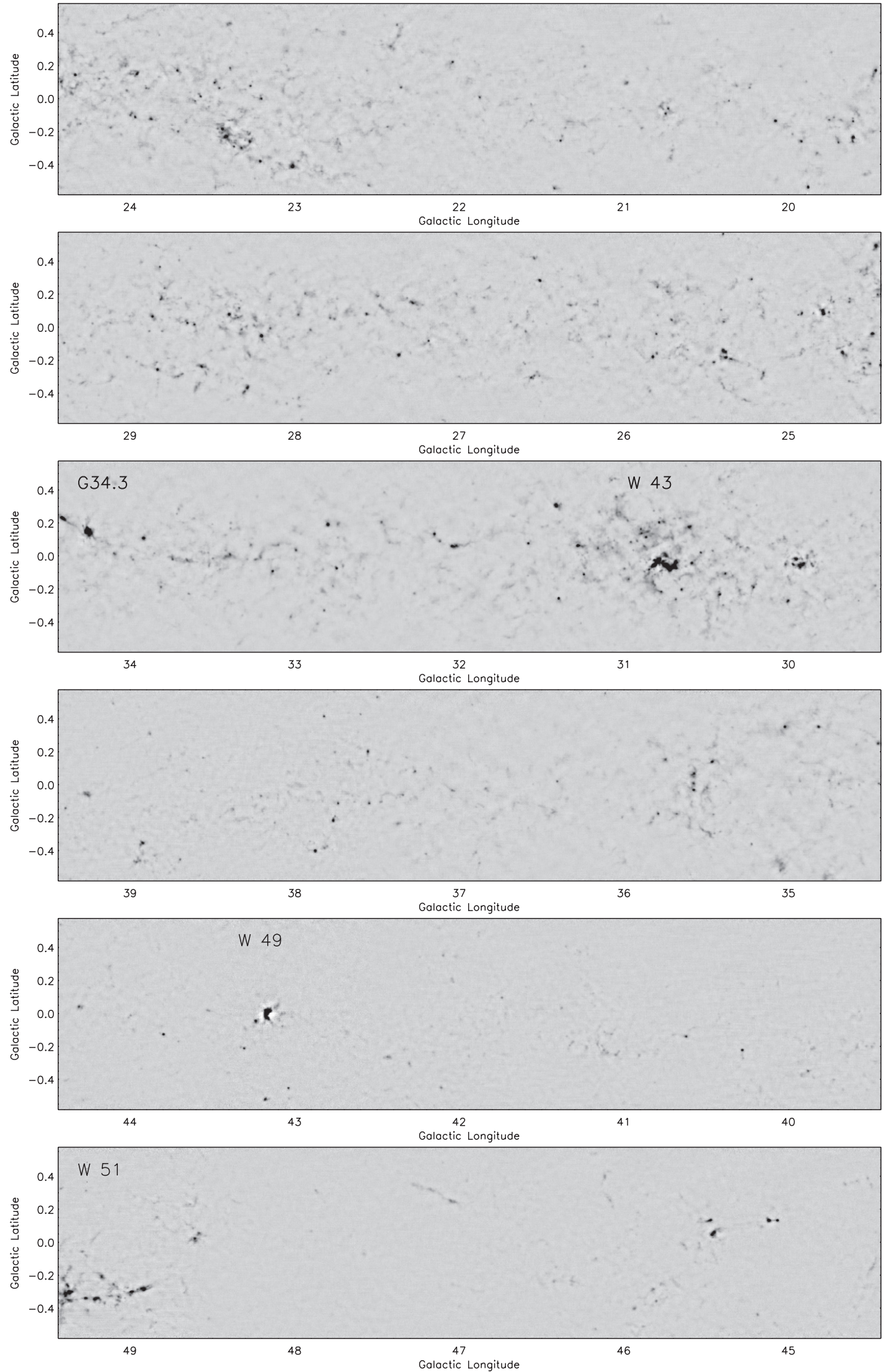

(b)

Figure 19. (Continued) 

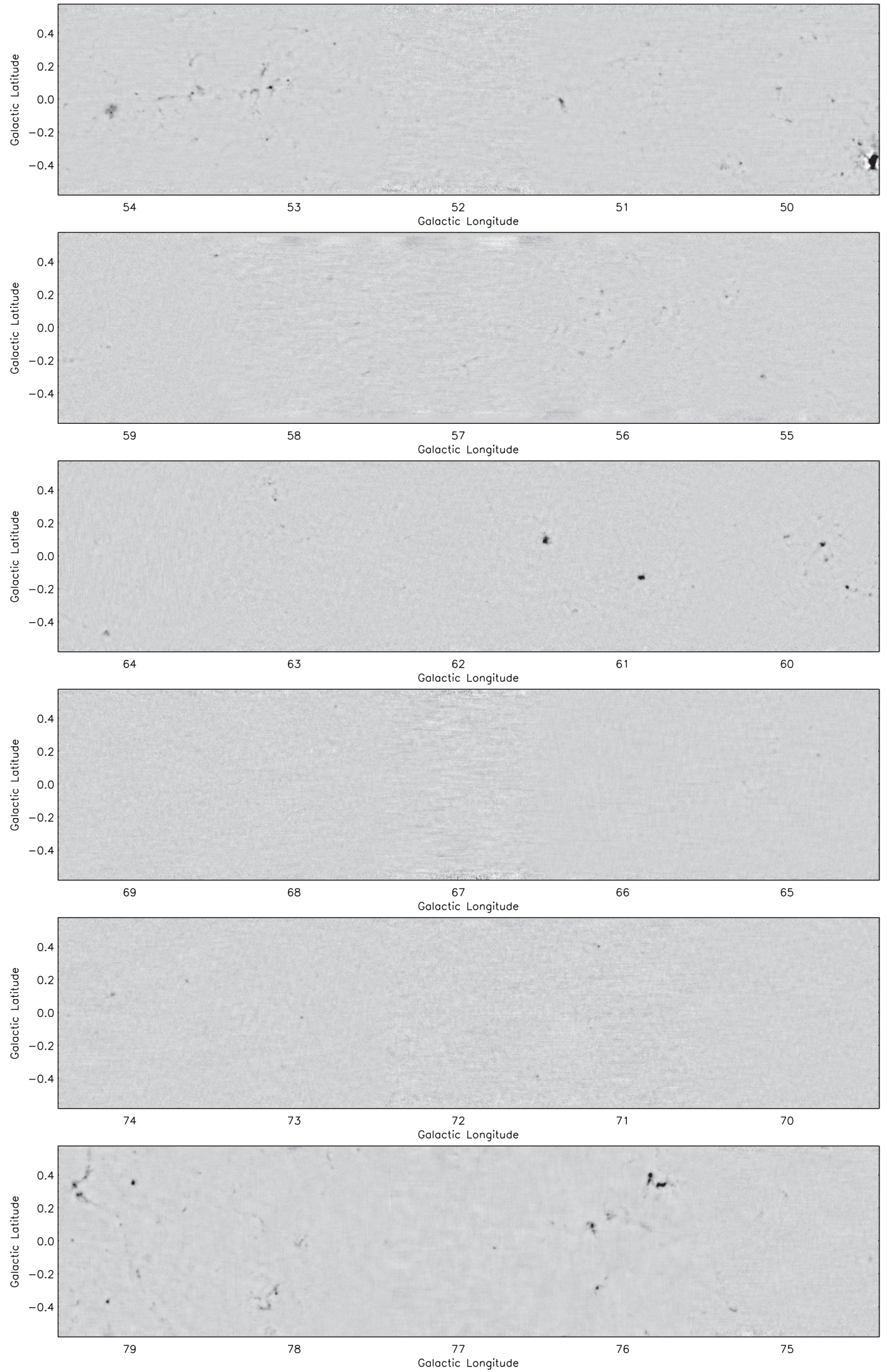

(c)

Figure 19. (Continued) 

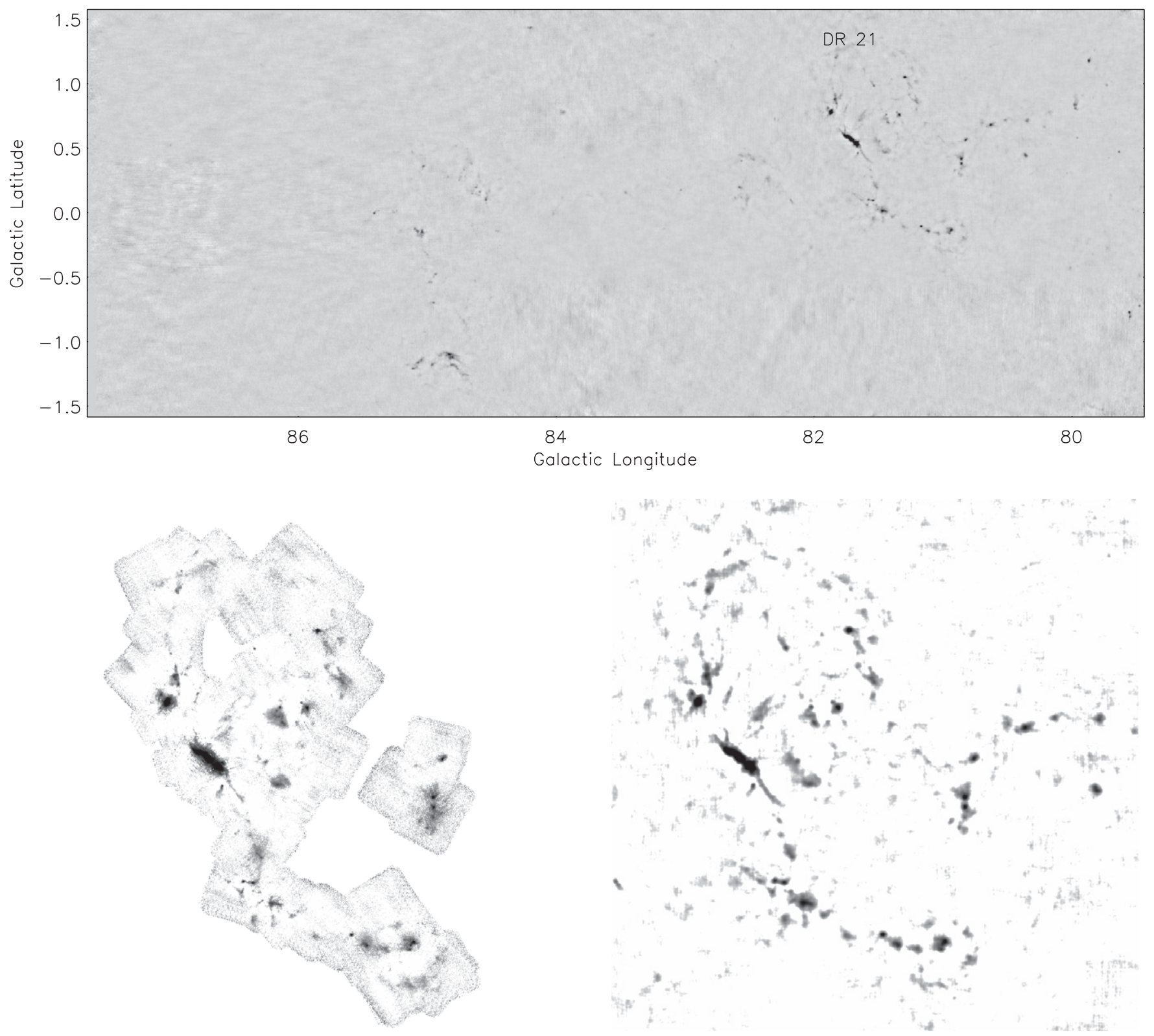

(b)

Figure 19. (Continued)

The Version 1.0 release includes the following images, each covering the same regions.

1. Calibrated maps for all regions observed (MAP). The maps are standard astrophysical maps subject to the caveats previously mentioned, the most important being the spatial filtering function (Section 4.6).

2. Maps of integration time per pixel (NHITSMAP). The nhitsmap shows how many recorded data points from the timestream have been assigned to each pixel; it is proportional to the dwell time per pixel (each "hit" is $0.1 \mathrm{~s}$ ).

3. Maps of the residual time series (NOISEMAP). The residual map is the map of the model timestream subtracted from the data timestream.

Examples of each are given in Figure 18. Future releases may contain additional data products.

\section{DISCUSSION}

The BGPS has observed a $170 \mathrm{deg}^{2}$ area at an effective resolution of a Gaussian PSF of 33" FWHM (corresponding to an area of $2.9 \times 10^{-8} \mathrm{sr}$ ). The positional uncertainty of the maps is $6^{\prime \prime}$ rms. The maps are calibrated into Jy beam ${ }^{-1}$ using Mars as a primary flux standard and accounting for atmosphere opacity variations in real time. The BGPS is well suited for studying source structure smaller than 3 arcmin in size. The processing of the maps removes more than $90 \%$ of flux for features with extents larger than 5.9, and attenuates the aperture flux of structures extending to 3.8 by $50 \%$. We have compared our flux densities to those of M07 and M09 and find that we need to multiply our flux densities up by a factor of 1.5 to match. We have investigated possible sources for the discrepancy, but do not understand the source of the difference. For now, we recommend multiplying the flux densities in the maps presented here and in 


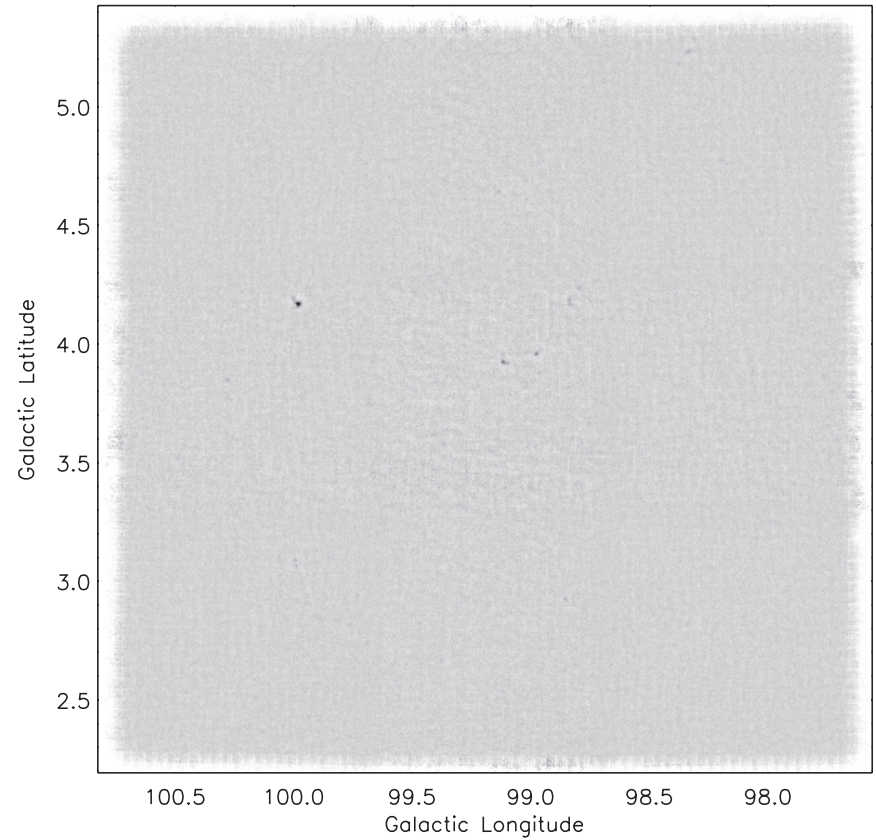

(e)

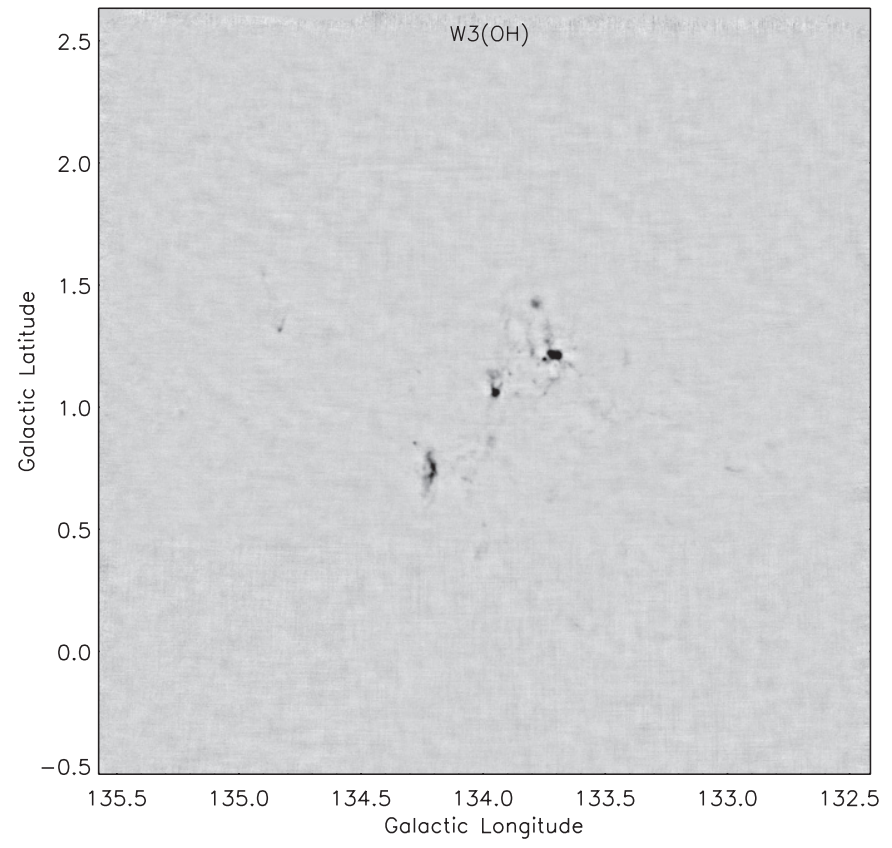

(g)

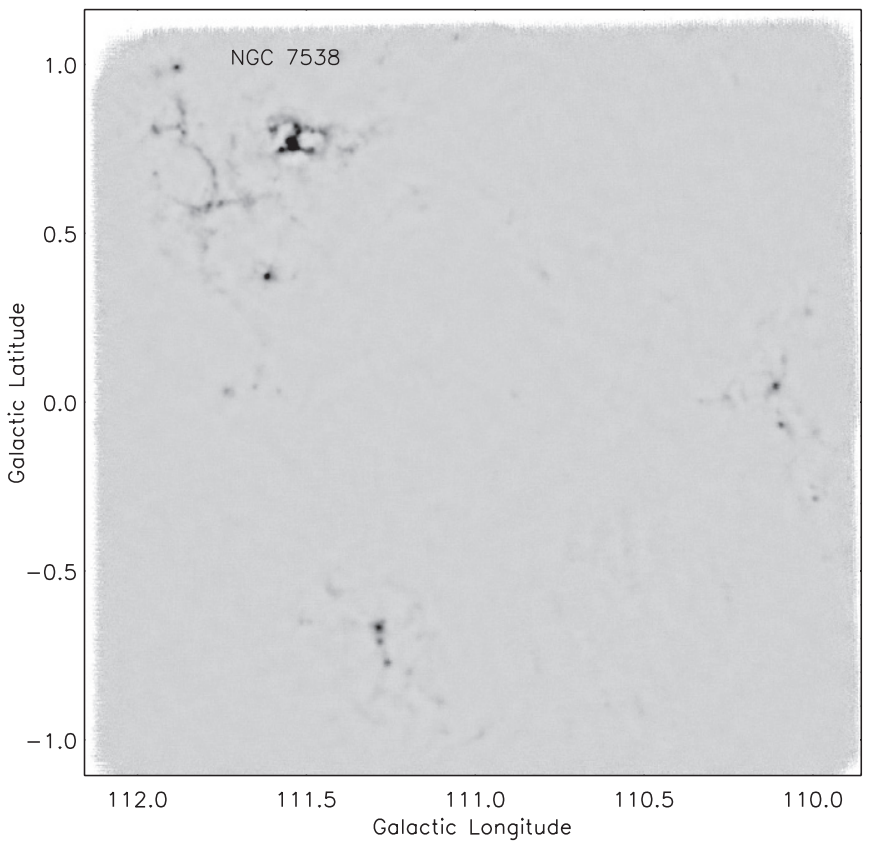

(f)

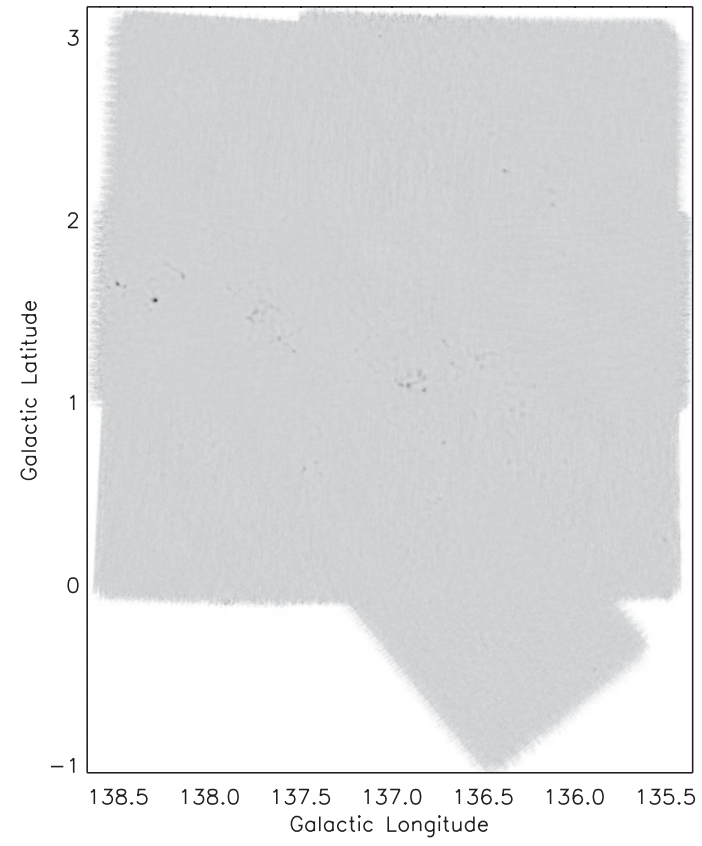

(h)

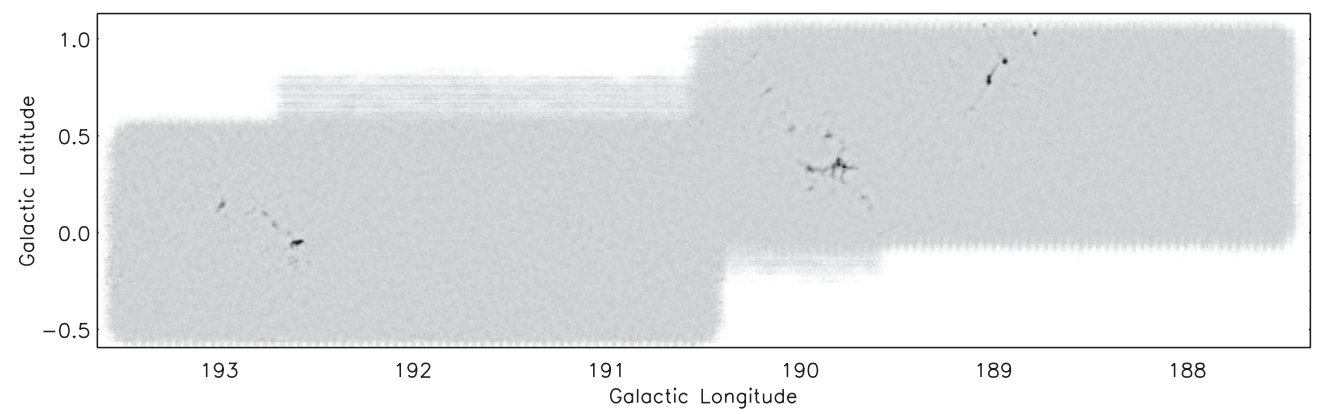

(i)

Figure 19. (Continued) 

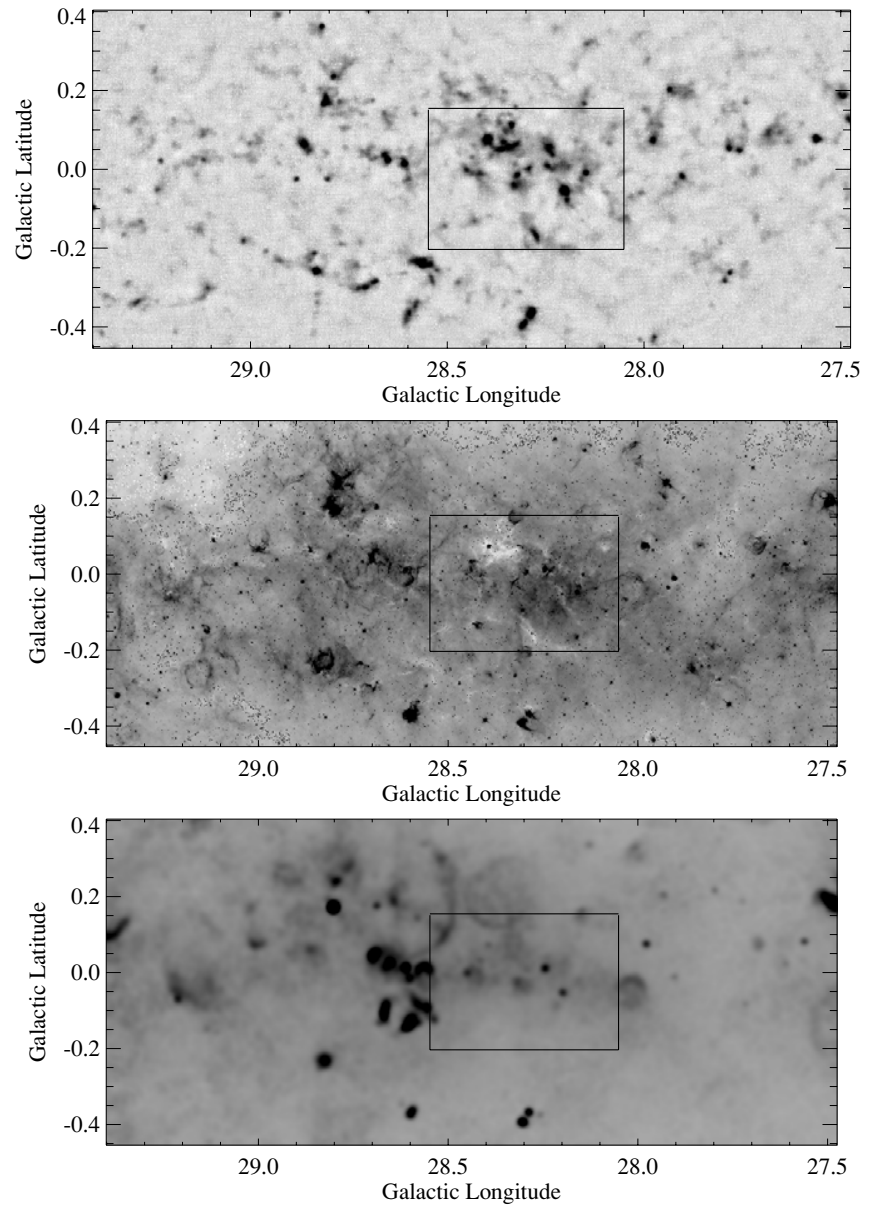

Figure 20. View of $2 \mathrm{deg}^{2}$ of the Galactic Plane centered at $l=28.45, b=0$. Top: BGPS, middle: Spitzer-GLIMPSE $8 \mu \mathrm{m}$ (1".9), and bottom: VGPS $20 \mathrm{~cm}$ continuum $\left(40^{\prime \prime}\right)$. IRDCs appear in the Spitzer negative image as wispy white regions against the gray emission background. While some of the Bolocam sources are apparent as IRDCs or strong infrared or radio sources, many lack any apparent association with features at these wavelengths. The box indicates the zoom-in region in Figures 21 and 22.

the R10 catalog by the factor $1.5 \pm 0.15$ to obtain consistency with other data sets. We are continuing to explore the source of this discrepancy, particularly in the calibration and filtering stages of the data pipeline. The latest data releases and updates on the discrepancy can be found on the BGPS IPAC Web site.

Maps from the entire BGPS are presented in Figure 19. Figures 5 and 19(d) show the generally excellent morphological agreement between BGPS maps and those at other (sub)millimeter wavelengths. Filamentary structures with aspect ratios up to the maximum spatial dynamic range of the survey $\left(354^{\prime \prime} / 33^{\prime \prime} \approx 10\right)$ are present throughout the Galactic plane. The most crowded fields consist of frothy and clumpy structure. The majority of sources are at least moderately resolved. We suspect that higher resolution observations will resolve BGPS clumps into clusters of protostellar objects, as has been seen in Serpens (Enoch et al. 2008; Testi \& Sargent 1998), OMC 1 (Beuther et al. 2004; Johnstone \& Bally 1999), and S255N (Cyganowski et al. 2007) where bolometer observations at low resolution have been complemented by interferometer observations at high resolution.

Figure 20 shows a comparison of the BGPS with SpitzerGLIMPSE $8 \mu \mathrm{m}$ (Benjamin et al. 2003) and VGPS $20 \mathrm{~cm}$ continuum images (Stil et al. 2006). In Figure 20, IRDCs appear

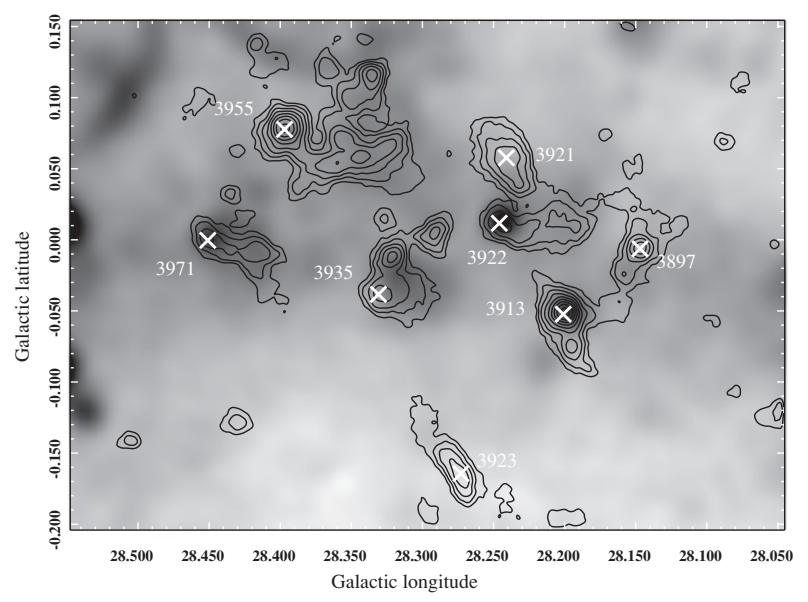

Figure 21. Zoom-in of the region shown in Figure 20, showing the VGPS image in inverted gray scale, with contours of BGPS emission in black. Selected sources from the R10 catalog are marked with X's and their catalog numbers. Note that while $\mathrm{H}$ II regions appear at or near the peaks of millimeter emission for clumps 3913, 3922, 3935, 3955, and 3971, others have no associated emission $(3897,3921,3923)$.

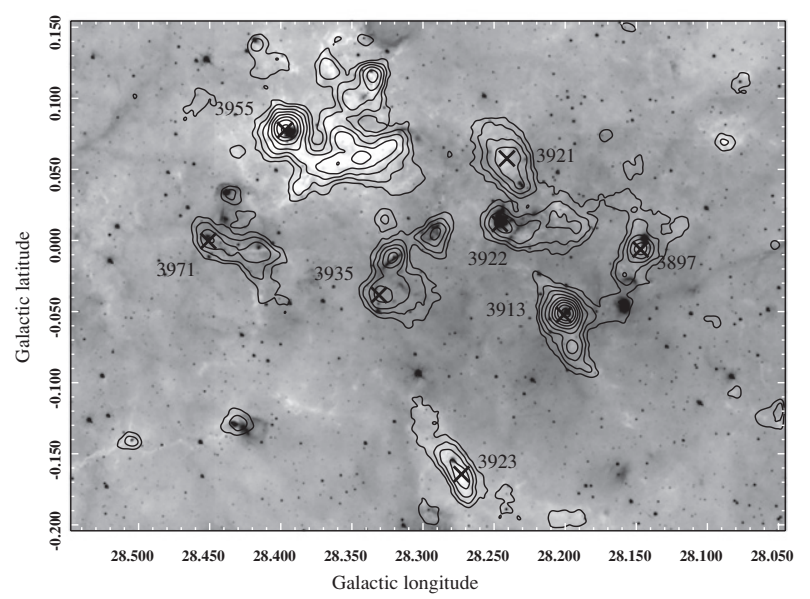

Figure 22. Zoom-in of the region shown in Figure 20, showing the SpitzerGLIMPSE image in inverted gray scale, with contours of BGPS emission in black. IRDCs appear as white in this image. Selected sources from the R10 catalog are marked with X's and their catalog numbers. Note the high degree of correspondence between the IRDC morphology and the millimeter emission for BGPS sources 3923 and 3955.

as the bright white clumps, often filamentary, near the midplane. BGPS sources are clearly associated with some of the H II regions detected by the VGPS; a zoom in of this region is shown in Figure 21. The comparison with $8 \mu \mathrm{m}$ IRDCs is particularly striking, with a high degree of correspondence in their morphologies. Detection of an IRDC requires that the cold, dense cloud be on the near-side of a bright mid-IR background, whereas BGPS sources are subject only to a sensitivity limit. Comparison of the GLIMPSE and BGPS data (see Figure 22) shows that some BGPS sources are clearly associated with IRDCs, a few are associated with IR-bright features (likely due to the association of free-free emission in the millimeter and PAH emission at $8 \mu \mathrm{m}$ ), and some have no clear IR counterpart. The BGPS sources which are neither bright nor dark at $8 \mu \mathrm{m}$ are probable candidates for "IRDC-like" clumps (cold, dense, potential proto-clusters) on the far-side or outer edges of the Galaxy. Thus, the BGPS could provide candidate pre-clusters independent of the favorable viewing angle of being on the near-side of bright mid-IR emission. An exploration of 


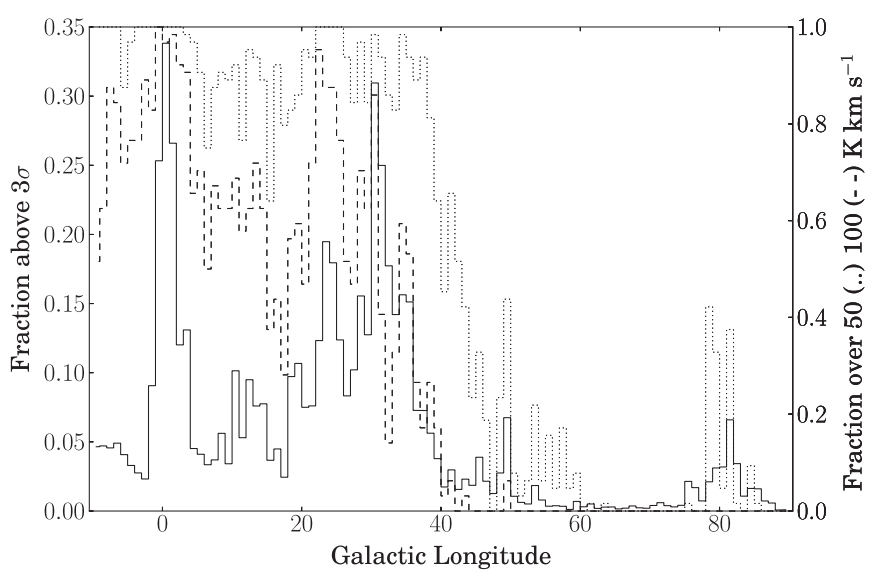

Figure 23. Filling factor of millimeter continuum emission from the BGPS compared to $\mathrm{CO}(1 \rightarrow 0)$ emission from Jackson et al. (2006). The axis on the left side gives the filling fraction above $3 \sigma$ of the local rms from the BGPS (solid line). The right axis gives the CO filling fraction above 50 (dotted) and 100 (dashed) $\mathrm{K} \mathrm{km} \mathrm{s}^{-1}$.

this population, as well as a detailed analysis of the physical properties and star formation tracers of IRDCs corresponding to BGPS sources, may be found in Battersby et al. (2010). It is worth noting that many features in the BGPS maps have no corresponding counterpart in the Spitzer or radio image.

There are clear maxima in the emission filling factor in the Galactic Center and in the region from $l=23$ to $l=31$, the line of sight through the densest part of the molecular ring. Additional areas of high source density are seen at $l=10$ and $l=13$, as shown in Figure 23. This result is in contrast to $\mathrm{CO}$ filling factors which are $\sim 1$ for $l \lesssim 40$ (Dame et al. 2001; Jackson et al. 2006). In parts of the plane where only one $\mathrm{CO}$ emission line is seen along any given line of sight $(l \gtrsim 40)$, the integrated emission is much lower, but there are still detections of $\mathrm{CO}$ in every pixel of the Dame et al. (2001) map in the latitude range $-0.5<b<0.5$. This behavior is consistent with the interpretation that the BGPS preferentially detects denser material than $\mathrm{CO}$. The connection of the millimeter emission with star-forming material awaits the detailed comparison to other star formation tracers. BGPS clumps are, however, associated with well-known star-forming regions: the brightest clumps visible in our maps are Sgr B2, G34.3+0.15, W 51, W 43, W 49, and M 17. These associations suggest that other bright sources of millimeter emission will prove to be massive star- or cluster-forming regions.

In some cases, the distance to BGPS clumps can be determined by matching Galactic Ring Survey ${ }^{13} \mathrm{CO}$ and $1.1 \mathrm{~mm}$ continuum morphology (Simon et al. 2006b; Jackson et al. 2006). However, in other cases the association between the $\mathrm{CO}$ and $1.1 \mathrm{~mm}$ data is not clear, either because of confusion or because the BGPS sources are too compact to identify in ${ }^{13} \mathrm{CO}$ morphology. Heterodyne follow-up observations using dense gas tracers
$\left(\mathrm{NH}_{3}, \mathrm{~N}_{2} \mathrm{H}^{+}, \mathrm{HCO}^{+}\right.$, and $\left.\mathrm{CS}\right)$ are being conducted to provide radial velocity, density, chemistry, and temperature measurements (W. Schlingman et al. 2011, in preparation; Dunham et al. 2010).

We have detected 8454 clumps in the surveyed fields (Rosolowsky et al. 2010), providing a sample of clumps suitable for multi-wavelength and high-resolution studies with existing telescopes and future facilities. This sample is free of the usual biases associated with observing only regions with signposts of star formation, such as $\mathrm{H}$ II regions or masers. The survey depth can be converted into an estimate of limiting mass sensitivity via standard estimates, i.e., under the assumption of a temperature, opacity, and distance. Using the dust opacity from Ossenkopf \& Henning $\left(1994 ; \kappa(1.1 \mathrm{~mm})=0.0114 \mathrm{~cm}^{2} \mathrm{~g}^{-1}\right)$, a gas-to-dust mass ratio of 100 , the Bolocam bandcenter from Table 4 , and the beam area as given in Section 5.1 the mass sensitivity can be written as

$$
M_{\mathrm{gas}} \approx 14.3\left(e^{13.0 / T_{d}}-1\right)\left(\frac{S_{v}}{1 \mathrm{Jy}}\right)\left(\frac{D}{1 \mathrm{kpc}}\right)^{2} M_{\odot} .
$$

At the coldest temperature expected $(10 \mathrm{~K})$, the mass increases by a factor of 2.9 from the mass assuming $20 \mathrm{~K}$. While sensitivity limits will bias the survey against distant, low-mass objects, initial results suggest that the Bolocam sources lie at a range of distances, and thus range from cores to clumps (Rosolowsky et al. 2010). We have begun to explore the variation of properties with Galactocentric radius with detailed analyses of the BGPS Galactic center (Bally et al. 2010) and anti-center (Dunham et al. 2010) data.

Millimeter-wavelength thermal dust emission reveals the repositories of the densest molecular gas, ranging in scale from cores to whole clouds. By pinpointing these regions, the BGPS allows the connection of this gas to nascent and ongoing star formation to be explored.

Facility: CSO (Bolocam)

We acknowledge the staff and day crew of the CSO for their assistance. The CSO is operated under NSF Cooperative Agreement AST-0838261. The BGPS project is supported by the National Science Foundation through NSF grant AST-0708403. J.A. was supported by a Jansky Fellowship from the National Radio Astronomy Observatory (NRAO). The first observing runs for BGPS were supported by travel funds provided by NRAO. Support for the development of Bolocam was provided by NSF grants AST-9980846 and AST-0206158. Team support was provided in part by NSF grant AST-0607793 to the University of Texas at Austin.

We recognize and acknowledge the cultural role and reverence that the summit of Mauna Kea has within the Hawaiian community. We are fortunate to conduct observations from this mountain.

\section{APPENDIX A}

\section{CALCULATION OF COLOR CORRECTIONS}

If an experiment has finite bandwidth $\left(t(v) \neq \delta\left(v-v_{c}\right)\right)$, to report a source surface brightness at a single frequency, one must assume a source spectrum. The power detected from that source is assumed to be

$$
P_{\mathrm{det}}=\eta A \Omega \int I_{0}(v) t(v) d v .
$$

(Here $\eta$ and $A \Omega$ are the optical efficiency and throughput of the instrument, $I_{0}(v)$ is the nominal (assumed) surface brightness of the 
source, and $t(v)$ is the bandpass transmission normalized to 1.0 at its peak.) Bolocam is single-moded, meaning that $A \Omega=\lambda^{2}$. The effective band center $v_{c}$ is defined implicitly by the equation

$$
I_{0}\left(v_{c}\right)=\frac{\int I_{0}(v) t(v) d v}{\int t(v) d v} .
$$

In calculating the color corrections and effective band centers, we consider two types of source spectra. The most physically motivated is a "graybody" spectrum parameterized as

$$
I_{\mathrm{GB}}(v)=\epsilon(v) B_{v}(T)=\left(1-\exp \left(-\tau_{v}\right)\right) B_{v}(T) \equiv\left(1-\exp \left[-\left(v / \nu_{0}\right)^{\beta}\right]\right) B_{v}(T),
$$

where $B_{v}(T)$ is the Planck function, $\epsilon(v)$ is the frequency-dependent emissivity, and $v_{0}$ is the frequency at which the optical depth $\tau_{v}$ reaches unity. For a frequency much lower than the blackbody peak and in the optically thin limit, this reduces to a simple power law

$$
I_{\alpha}(v)=\left(\frac{v}{v_{0}}\right)^{\beta} \frac{2 k T v^{2}}{c^{2}} \propto\left(\frac{v}{v_{0}}\right)^{\alpha}
$$

The color correction $K$ is defined by the relative change in the intensity which would be measured at the same effective band center if the source had a different spectrum $I_{1}(v)$ than that assumed for calculating the band center; thus,

$$
\begin{aligned}
I_{1}\left(v_{c}\right) & =I_{0}\left(v_{c}\right) \frac{I_{1}\left(v_{c}\right)}{\int I_{1}(v) t(v) d v}\left[\frac{I_{0}\left(v_{c}\right)}{\int I_{0}(v) t(v) d v}\right]^{-1} \\
& \equiv K^{-1} I_{0}\left(v_{c}\right) .
\end{aligned}
$$

We calculate the effective band centers and color corrections for Bolocam in Table 4. The fiducial spectrum for quoting the bandcenter (chosen because of its closeness to mean Galactic properties; e.g., Reach et al. 1995) is taken to be that of Equation (A3) with parameter values $T=20 \mathrm{~K}, \beta=1.8$, and $\nu_{0}=3000 \mathrm{GHz}(100 \mu \mathrm{m})$. The bandcenters and color corrections relative to this fiducial spectrum are also computed for various values of $\alpha$ in Equation (A4). Over the frequency range of the Bolocam passband, a power law with $\alpha=3.5$ is a very good approximation to the assumed graybody.

\section{APPENDIX B}

\section{FITS HEADER INFORMATION}

Below is a sample FITS header from the version 1.0.2 release of the BGPS images described in this paper.

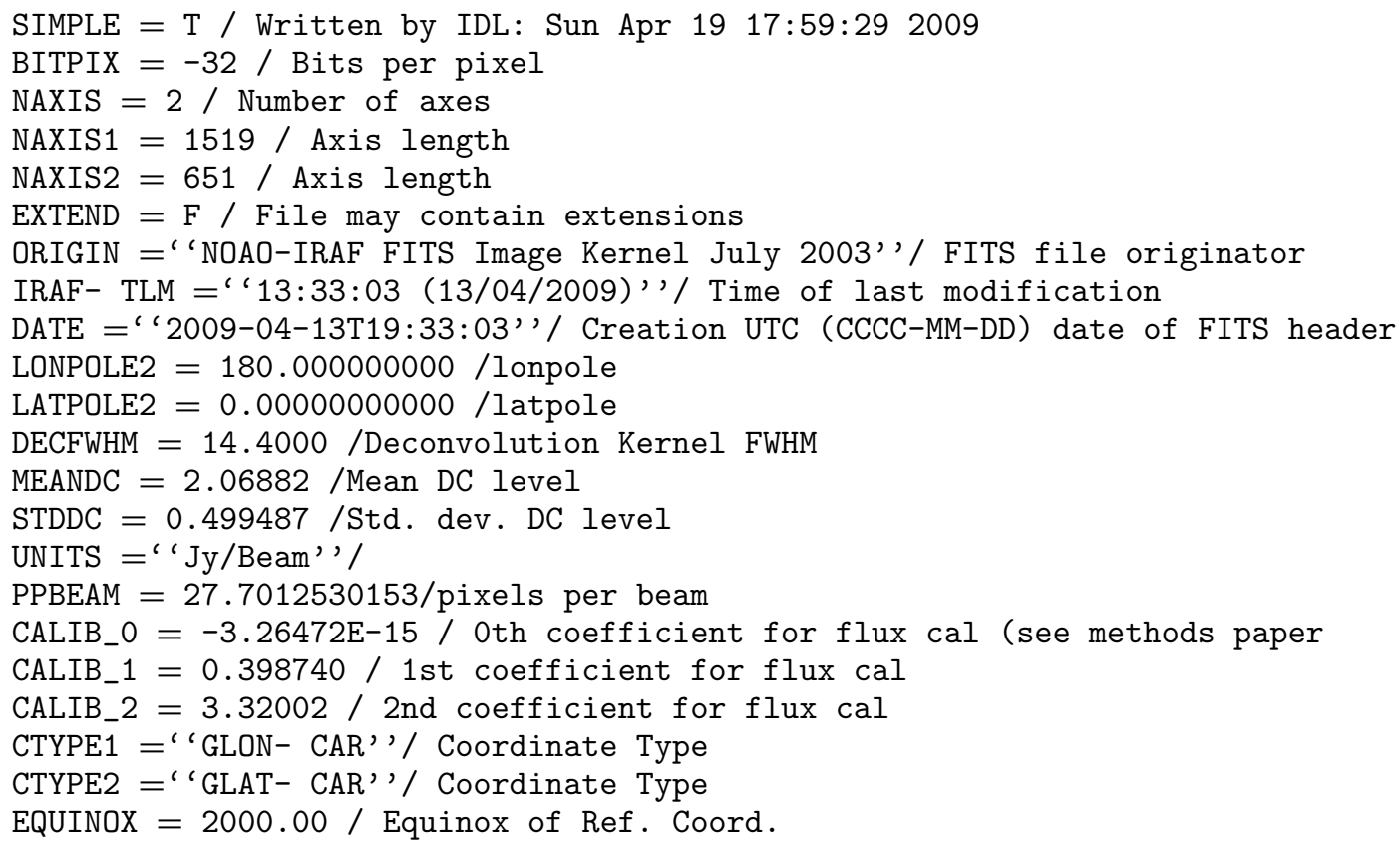




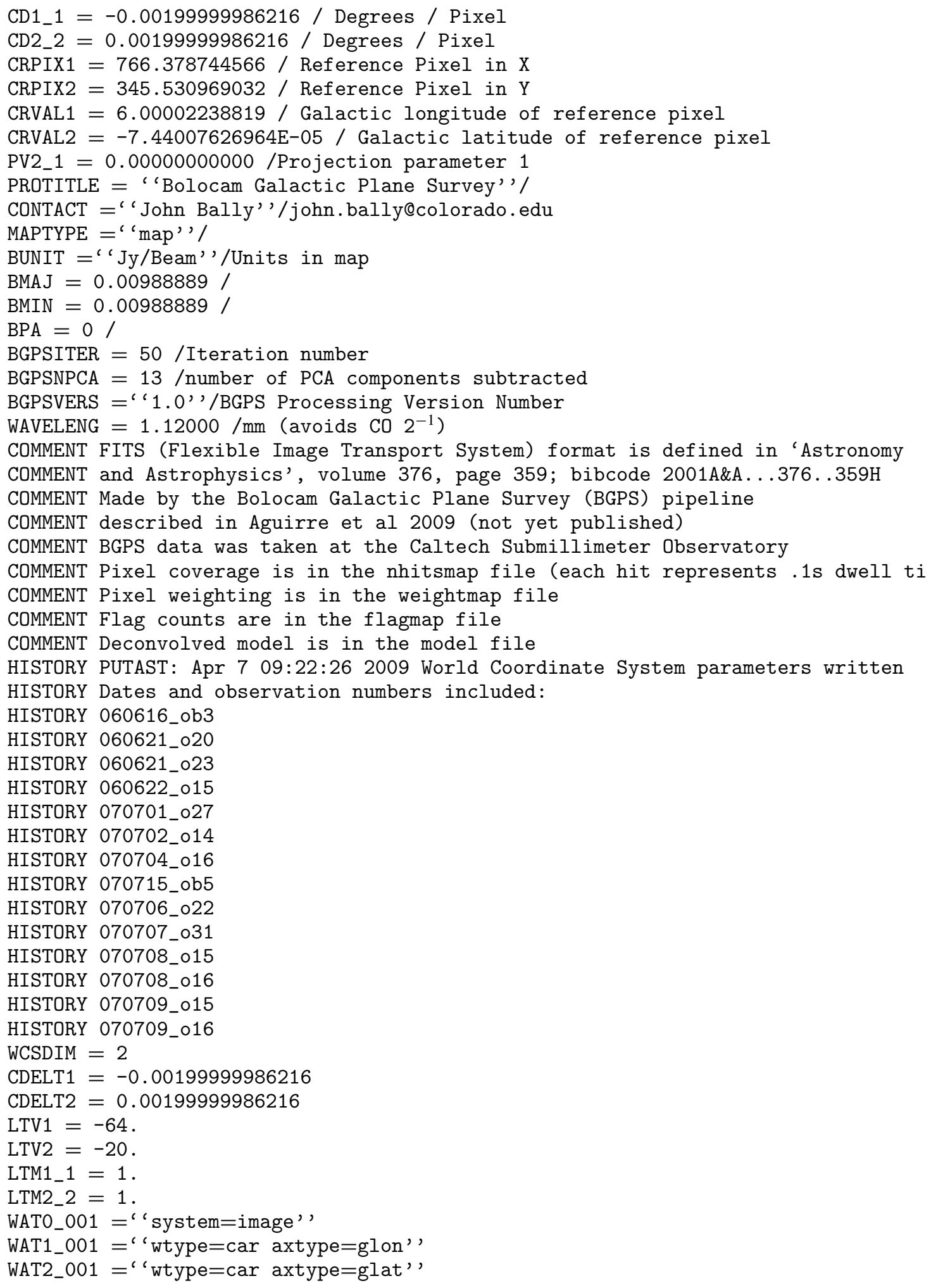




\section{REFERENCES}

Bally, J., et al. 2010, ApJ, 721, 137

Battersby, C., Bally, J., Jackson, J. M., Ginsburg, A., Shirley, Y. L., Schlingman, W., \& Glenn, J. 2010, ApJ, 721, 222

Benjamin, R. A., et al. 2003, PASP, 115, 953

Beuther, H., et al. 2004, ApJ, 616, L31

Buckle, J. V., et al. 2009, MNRAS, 399, 1026

Carey, S. J., Clark, F. O., Egan, M. P., Price, S. D., Shipman, R. F., \& Kuchar, T. A. 1998, ApJ, 508, 721

Carey, S. J., et al. 2009, PASP, 121, 76

Chapin, E. L., et al. 2008, ApJ, 681, 428

Cotton, W. D., et al. 2009, ApJ, 701, 1872

Cyganowski, C. J., Brogan, C. L., \& Hunter, T. R. 2007, AJ, 134, 346

Dame, T. M., Hartmann, D., \& Thaddeus, P. 2001, ApJ, 547, 792

Di Francesco, J. 2008, BAAS, 40, 271

Di Francesco, J., Johnstone, D., Kirk, H., MacKenzie, T., \& Ledwosinska, E. 2008, ApJS, 175, 277

Dunham, M. K., et al. 2010, ApJ, 717, 1157

Egan, M. P., Shipman, R. F., Price, S. D., Carey, S. J., Clark, F. O., \& Cohen, M. 1998, ApJ, 494, L199

Enoch, M. L., Evans, N. J., II, Sargent, A. I., Glenn, J., Rosolowsky, E., \& Myers, P. 2008, ApJ, 684, 1240

Enoch, M. L., Glenn, J., Evans, N. J., II, Sargent, A. I., Young, K. E., \& Huard, T. L. 2007, ApJ, 666, 982

Enoch, M. L., et al. 2006, ApJ, 638, 293

Evans, N. J., et al. 2009, ApJS, 181, 321

Glenn, J., et al. 2003, Proc. SPIE, 4855, 30

Griffin, M. J., \& Orton, G. S. 1993, Icarus, 105, 537

Holland, W. S., et al. 1999, MNRAS, 303, 659

Hollis, J. M., Dorband, J. E., \& Yusef-Zadeh, F. 1992, ApJ, 386, 293

Jackson, J. M., et al. 2006, ApJS, 163, 145

Johnstone, D., \& Bally, J. 1999, ApJ, 510, L49

Johnstone, D., \& Bally, J. 2006, ApJ, 653, 383

Kauffmann, J., Bertoldi, F., Bourke, T. L., Evans, N. J., II, \& Lee, C. W. 2008, A\&A, 487, 993

Kovács, A. 2008, Proc. SPIE, 7020, 70201S

Lada, E. A., Bally, J., \& Stark, A. A. 1991, ApJ, 368, 432

Laurent, G. T., et al. 2005, ApJ, 623, 742
Matthews, H., et al. 2009, AJ, 138, 1380

McKee, C. F., \& Ostriker, E. C. 2007, ARA\&A, 45, 565

Molinari, S., et al. 2010, PASP, 122, 314

Motte, F., \& André, P. 2001, A\&A, 365, 440

Motte, F., Bontemps, S., Schilke, P., Schneider, N., Menten, K. M., \& Broguière, D. 2007, A\&A, 476, 1243

Mueller, K. E., Shirley, Y. L., Evans, N. J., II, \& Jacobson, H. R. 2002, ApJS, 143,469

Netterfield, C. B., et al. 2009, ApJ, 707, 1824

Nummelin, A., Bergman, P., Hjalmarson, A., Friberg, P., Irvine, W. M., Millar, T. J., Ohishi, M., \& Saito, S. 1998, ApJS, 117, 427

Olmi, L., et al. 2009, ApJ, 707, 1836

Orton, G. S., Griffin, M. J., Ade, P. A. R., Nolt, I. G., \& Radostitz, J. V. 1986, Icarus, 67, 289

Ossenkopf, V., \& Henning, T. 1994, A\&A, 291, 943

Peretto, N., \& Fuller, G. A. 2009, A\&A, 505, 405

Pohl, M., Englmaier, P., \& Bissantz, N. 2008, ApJ, 677, 283

Rathborne, J. M., Jackson, J. M., \& Simon, R. 2006, ApJ, 641, 389

Rathborne, J. M., Jackson, J. M., Zhang, Q., \& Simon, R. 2008, ApJ, 689, 1141

Reach, W. T., et al. 1995, ApJ, 451, 188

Reid, M. J., et al. 2009, ApJ, 700, 137

Rosolowsky, E., et al. 2010, ApJS, 188, 123

Roy, A., et al. 2010, ApJ, 708, 1611

Sayers, J., et al. 2009, ApJ, 690, 1597

Sayers, J., et al. 2010, ApJ, 708, 1674

Schuller, F., et al. 2009, A\&A, 504, 415

Simon, R., Jackson, J. M., Rathborne, J. M., \& Chambers, E. T. 2006a, ApJ, 639,227

Simon, R., Rathborne, J. M., Shah, R. Y., Jackson, J. M., \& Chambers, E. T. 2006b, ApJ, 653, 1325

Stark, A. A., \& Lee, Y. 2006, ApJ, 641, L113

Stil, J. M., et al. 2006, AJ, 132, 1158

Testi, L., \& Sargent, A. I. 1998, ApJ, 508, L91

van Dishoeck, E. F., \& Blake, G. A. 1998, ARA\&A, 36, 317

Williams, J. P., Blitz, L., \& McKee, C. F. 2000, in Protostars and Planets IV, ed. V. Mannings, A. P. Boss, \& S. S. Russell (Tucson, AZ: Univ. Arizona Press), 97

Young, K. E., et al. 2006, ApJ, 644, 326 